\title{
Publicación Electrónica
}

ASOCIACIÓN PALEONTOLÓGICA ARGENTINA

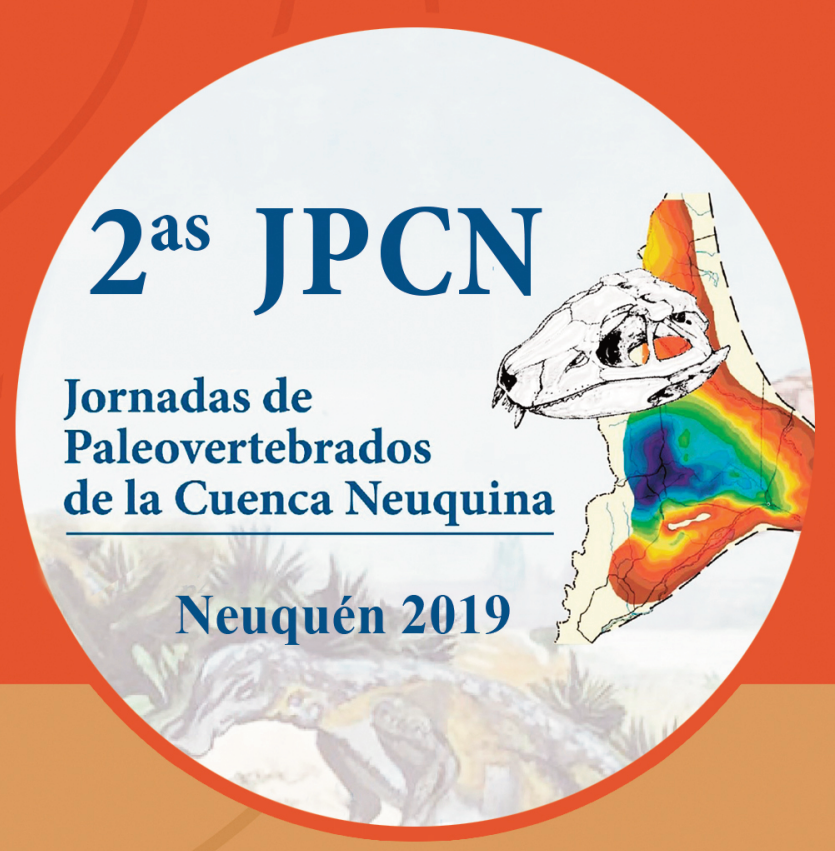

\section{LIBRO DE RESÚMENES}

31 de Octubre al 02 de Noviembre de 2019

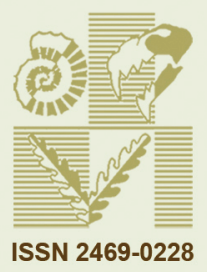




\section{Libro de Resúmenes}
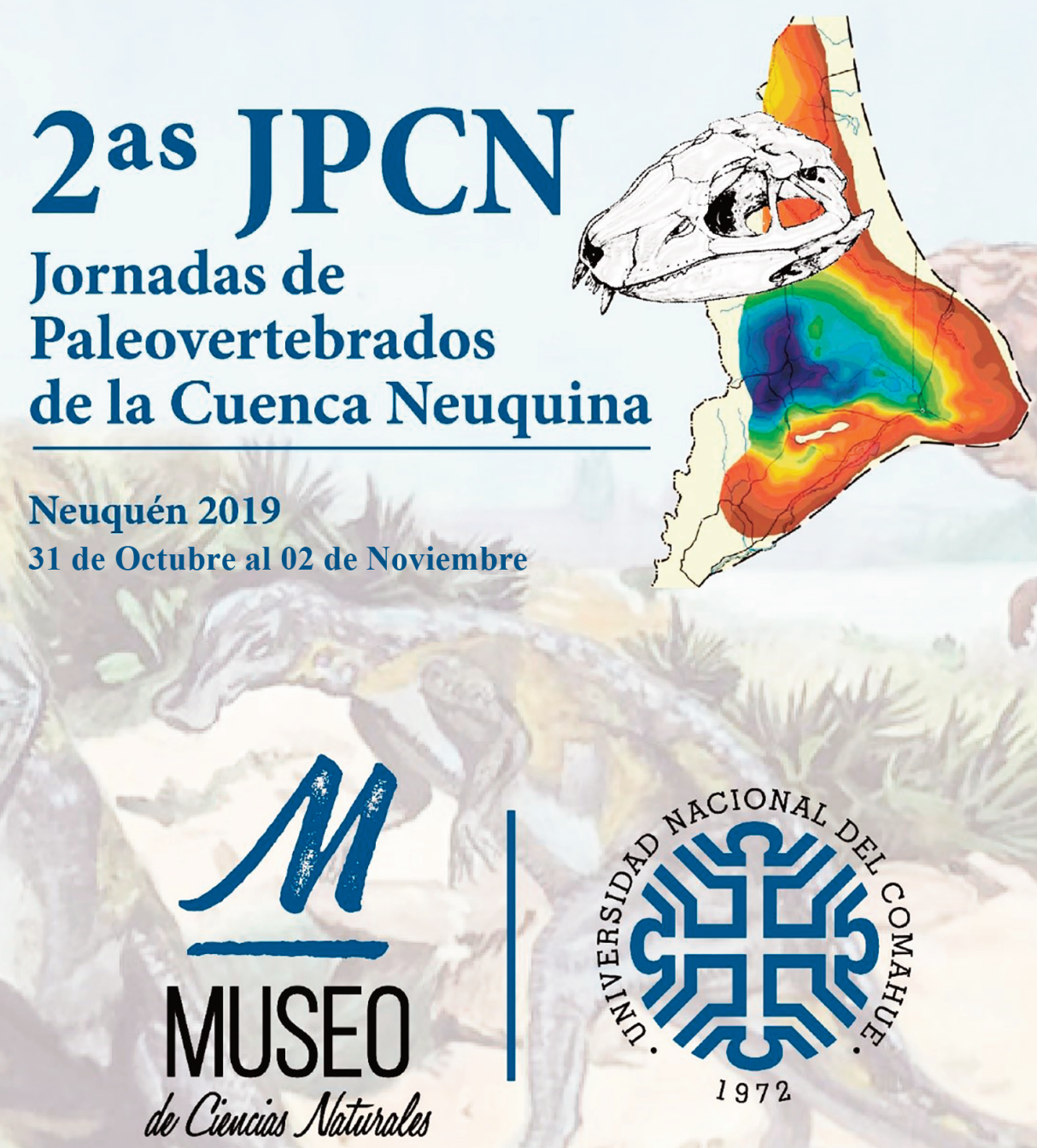

MINISTERIO DE LAS CULTURAS 


\section{Instituciones que brindan su aval}
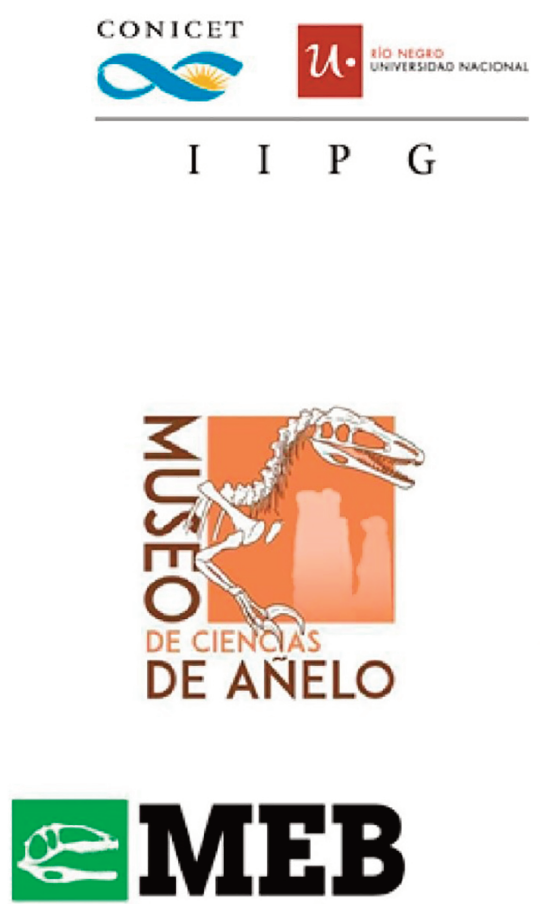

Museo Paleontológico

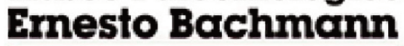

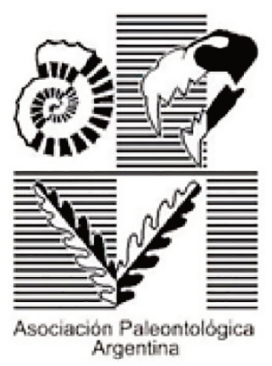

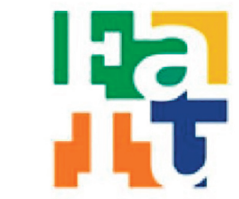

Facultad de

TURISMO

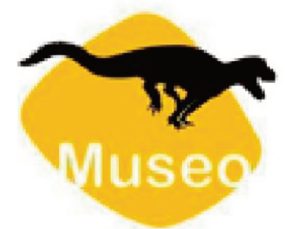

Ciencias Naturales Municipsildsd de Senillons

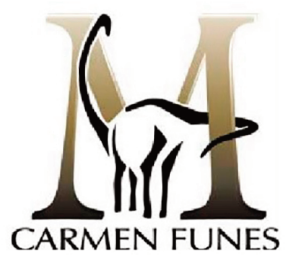

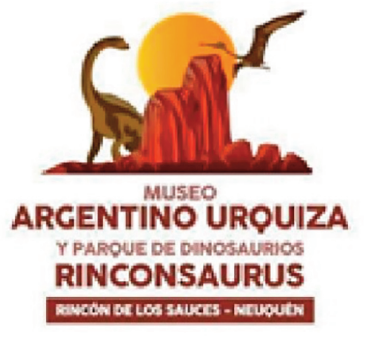

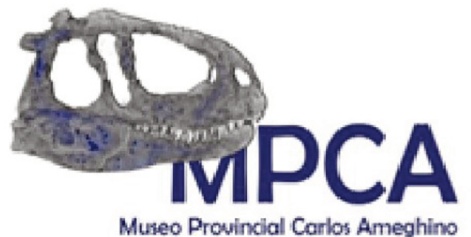

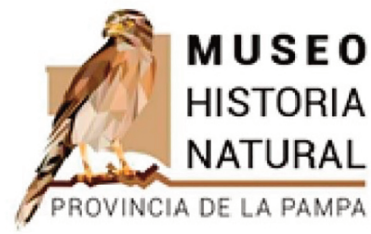
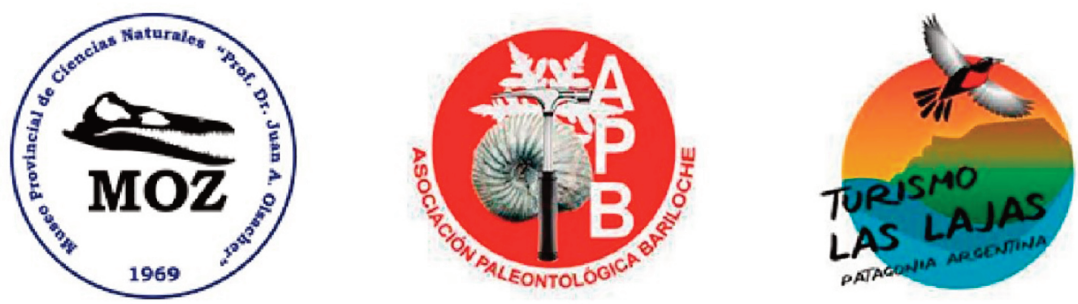

MINISTERIO

NaUgúa

PROVINCIA
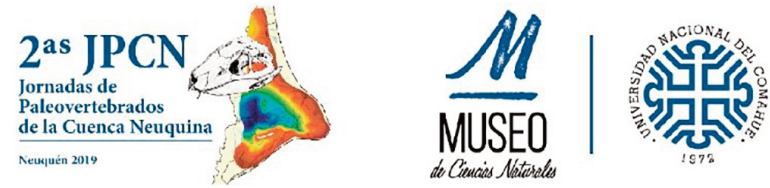


\title{
LIBRO DE RESÚMENES
}

\section{$2^{\text {as }} \mathrm{JPCN}$ \\ Jornadas de Paleovertebrados de la Cuenca Neuquina}

31 de Octubre al 02 de Noviembre, Neuquén

\section{COMISIÓN ORGANIZADORA}

\author{
Juan D. Porfiri \\ Museo Ciencias Naturales y Facultad de Ingeniería de la Universidad Nacional del Comahue, Neuquén. \\ Museo de Ciencias de Añelo, Neuquén. \\ Mattia A. Baiano \\ Museo Municipal Carmen Funes, Plaza Huincul, Neuquén. \\ Instituto de Investigación en Paleobiología y Geología - CONICET Universidad Nacional de Río Negro. \\ Flavio Bellardini \\ Dirección Provincial de Patrimonio Cultural, Neuquén. \\ Domenica D. Dos Santos \\ Museo Ciencias Naturales y Facultad de Ingeniería de la Universidad Nacional del Comahue, Neuquén. \\ Museo de Ciencias de Añelo, Neuquén.
}

Leonardo S. Filippi

Museo Municipal Argentino Urquiza, Rincón De Los Sauces, Neuquén.

Alberto C. Garrido

Museo Provincial de Ciencias Naturales Prof. Dr. Juan A. Olsacher, Zapala, Neuquén. Dirección Provincial de Minería. Centro de Investigación en Geociencias de la Patagonia Dpto. de Geología y Petróleo.

Fain Universidad Nacional del Comahue.

Javier Santoni

Subsecretaría de Vinculación y Transferencia Universidad Nacional del Comahue.

Rubén D. Juárez Valieri

Secretaria de Cultura de la Provincia de Río Negro. Museo Provincial Carlos Ameghino, Cipolletti, Río Negro.

Museo de Ciencias de Añelo, Neuquén.

Yusara Mastrocola

Facultad de Turismo Universidad Nacional del Comahue.

Jorge G. Meso

Instituto de Investigación en Paleobiología y Geología - CONCIET Universidad Nacional de Río Negro

Magdalena Perini

Facultad de Turismo Universidad Nacional del Comahue Copade - Neuquén.

Luisa E. Villar

Municipalidad de General Roca, Río Negro.

Guillermo Windholz

Museo Municipal Carmen Funes, Plaza Huincul, Neuquén.

Instituto de Investigación en Paleobiología y Geología - CONICET Universidad Nacional de Río Negro.

Gabriela Dupen

Facultad de Turismo Universidad Nacional del Comahue. 


\section{COMITÉ CIENTíFICO}

Francisco Barrios

Museo Provincial de Ciencias Naturales Prof. Dr. Juan A. Olsacher, Zapala, Neuquén.

José L. Carballido

Museo Egidio Feruglio, CONICET, Chubut.

Laura Codorniú

CONICET, Dpto. de Geología Universidad Nacional de San Luis.

Laura E. Cruz

Museo Argentino de Ciencias Naturales Bernardino Rivadavia - CONICET.

Claudia Della Negra

Dirección Provincial de Patrimonio Cultural, Neuquén.

Lucas E. Fiorelli

Centro Regional de Investigaciones Científicas y Transferencia Tecnológica de La Rioja (CRILAR) - CONICET

Romina González

Centro de Ecología Aplicada del Litoral (CECOAL), Corrientes.

Noemí Gutierrez

Facultad de Turismo Universidad Nacional del Comahue.

Arturo M. Heredia

CONICET-IDEAN, Universidad De Bs. As.

Agustina Lecuona

CONICET-IIPG-UNRN.

Juan M. Janello

IANIGLA-CONICET. Museo de Historia Natural de San Rafael, Mendoza, Argentina.

Yusara Mastrocola

Universidad Nacional del Comahue Facultad de Turismo.

Ariel H. Méndez

Instituto Patagónico de Geología y Paleontología (IPGP), CENPAT, Chubut -CONICET.

Matías Motta

Laboratorio de Anatomía Comparada y Evolución de los Vertebrados, Museo Argentino de Ciencias Naturales

"Bernardino Rivadavia", CABA, Argentina.

Karen M. Panzeri

Museo de la Plata, CONICET.

Mauro Passalia

CONICET-INIBIOMA - Universidad Nacional del Comahue, Bariloche.

M. Magdalena Perini

Facultad de Turismo Universidad Nacional del Comahue. COPADE, Neuquén.

Elena Previtera

Instituto Argentino de Nivología, Glaciología y Ciencias Ambientales (IANIGLA), CCT-CONICET - Mendoza, Argentina.

Sebastián Rozadilla

Laboratorio de Anatomía Comparada y Evolución de los Vertebrados, Museo Argentino de Ciencias Naturales

"Bernardino Rivadavia", CABA, Argentina.

Sandra Sánchez

Facultad de Turismo Universidad Nacional del Comahue.

M. Belén Tomaselli

Laboratorio y Museo de Dinosaurios, ICB/CONICET.

Luisa E. Villar

Municipalidad de General Roca, Río Negro.

\section{COLABORADORES}

Liliana Berra, Martín Gasparini, Darío López, Macarena Martínez, Nasib Neme, Santiago Sánchez, Cecilia Valderrama, Julieta Páez

Universidad Nacional del Comahue

Damiano Palombi

Universidad Nacional de Río Negro

doi: 10.5710/PEAPA.07.07.2020.329 


\title{
SECRETOS GUARDADOS BAJO LA PIEL: SOBRE LA MICROESTRUCTURA ÓSEA DE LOS OSTEODERMOS EN TETRÁPODOS Y SU VALOR EN PALEOBIOLOGÍA Y SISTEMÁTICA
}

\author{
I. A. CERDA ${ }^{1}$
}

${ }^{1}$ CONICET, Instituto de Investigación en Paleobiología y Geología (IIPG-UNRN), Museo Provincial "Carlos Ameghino". Belgrano 1700, Paraje Pichi Ruca (predio Marabunta), 8300 Cipolletti, Río Negro, Argentina.nachocerda6@yahoo.com.ar

Los osteodermos son estructuras óseas formadas a nivel de la dermis en numerosos grupos de tetrápodos, incluyendo formas tanto fósiles como vivientes. El estudio de la anatomía e histología de estos elementos ha brindado información relevante no solo a nivel sistemático, sino también paleobiológico. En el campo de la paleohistología, la presencia y organización de determinados tejidos/estructuras permite inferir el origen e histogénesis de los osteodermos y establecer los mecanismos involucrados en la formación y mantenimiento de la ornamentación superficial de estas estructuras, la cual es característica de varios grupos de tetrápodos. Del mismo modo, el número de marcas de crecimiento (i.e., Líneas de Crecimiento Detenido y annuli) se utiliza comúnmente para deducir la edad relativa o absoluta de los individuos (i.e., esqueletocronología). Considerando el origen e histogénesis de los osteodermos, los dos mecanismos principales de osificación (intramembranosa y metaplásica) son inferidos a partir de la presencia y distribución de fibras mineralizadas de la dermis. Con respecto a la formación y mantenimiento de la ornamentación superficial, se han descripto dos mecanismos básicos basados en: crecimiento diferencial del tejido óseo y reabsorción y nueva formación de tejido óseo. Con respecto a los estudios esqueletocronológicos, al menos dentro de ciertos linajes, los osteodermos demuestran ser más efectivos para estimar edades que otros tipos de elementos (e.g., costillas, huesos apendiculares). La posibilidad de inferir edades a partir de la histología de los osteodermos ha permitido reconstruir curvas de crecimiento en algunas pocas especies.

\section{UN NEUQUINO EN TIERRAS INCAS: RESTOS AFINES A NEUQUENSAURUS DEL CRETÁCICO DE ECUADOR}

\author{
S. Apesteguía 1,2, J. E. Soto Luzuriaga³, P. A. Gallina ${ }^{1,2}$, J. Tamay Granda ${ }^{3}$ y G. A. Guamán Jaramillo ${ }^{3}$ \\ ${ }^{1}$ CONICET \\ 2'Área de Paleontología, Fundación de Historia Natural Félix de Azara, Universidad Maimónides. Hidalgo 775, 1405 Ciudad Autónoma de \\ Buenos Aires, Argentina. sebapesteguia@gmail.com \\ ${ }^{3}$ Departamento de Geología y Minas e Ingeniería Civil, Universidad Técnica Particular de Loja. San Cayetano Alto, Apartado Postal 11-01-608, \\ Ecuador.jesoto@utpl.edu.ec; jvtamay@utpl.edu.ec; gaguaman2@utpl.edu.ec
}

Restos de un nuevo titanosaurio (Saurischia, Sauropoda) han sido colectados en rocas del Cretácico Superior de la Formación Río Playas (Campaniano-Maastrichtiano) de la Cuenca Alamor-Lancones, del sur de Ecuador. Los restos incluyen parte de un sacro (YM-UTPL_002), de una vértebra caudal media (YM-INPC-014), y varios huesos apendiculares incompletos (YMINPC-015-17). El espécimen muestra en su vértebra caudal anterior a media el cóndilo comprimido dorsoventralmente, con el extremo posterior elevado con respecto a la línea media, sin cresta longitudinal ventral, y estructura interna esponjosa desprovista de grandes cavidades (i.e., hueso camelado, compartido con Neuquensaurus). Además, el último centro sacro resulta tan largo como alto, con una fosa pequeña somera y de abertura ovoide sobre la cara lateral. De entre los huesos apendiculares, el radio es robusto, de diáfisis aplanada y con un cíngulo marcado justo bajo la epífisis, con una superficie proximal de contorno heptagonal. La morfología y tamaño del espécimen muestran que el titanosaurio hallado en Ecuador pertenece a una especie muy cercanamente relacionada a Neuquensaurus, además de ser de una antigüedad coincidente. Se trata, además, del saltasaurino más boreal registrado hasta el momento. 


\title{
REVISITING VELOCISAURUS UNICUS BONAPARTE, 1991 (THEROPODA, CERATOSAURIA, NOASAURIDAE): INFERENCES ON THE NOASAURIDAE/ABELISAURIDAE SYSTEMATIC
}

\author{
M. A. BAIANO ${ }^{1,2}$ AND R. A. CORIA ${ }^{1}$
}

1CONICET-Museo Municipal "Carmen Funes". Av. Córdoba 55, 8318 Plaza Huincul, Neuquén, Argentina. mbaiano@unrn.edu.ar ${ }^{2}$ CONICET-IIPG-UNRN. Av. Roca 1242, 8332 General Roca, Río Negro, Argentina.

In 1991, Bonaparte described Velocisaurus unicus upon an almost complete right tibia and a near complete right autopodium. He regarded this specimen a new taxon on the base of a metatarsal II and IV very mediolaterally compressed, being the metatarsal III two to three times wider than metatarsals II and IV. Brusatte and Carrano considered this taxon devoid of peculiar characters, although they kept it valid on the base of its provenance. In contrast, Brisson and colleagues reported a new specimen of this species and reinforced its validity by identifying three new autapomorphies. Indeed, all Velocisaurus's autapomorphies cited by the aforementioned authors seem to have a rather wide distribution within Noasauridae, or even they are convergent with Abelisauridae. For instance, a triangular transverse section of the proximal end of the femur (hypertrophy of anterior muscular line) is present also in the abelisaurids Ekrixinatosaurus, Xenotarsosaurus, Skorpiovenator and Aucasaurus; the presence of transversely thin metatarsals II and IV are typical of noasaurids, however, the rod-like shape of metatarsals II mentioned by Brisson and colleagues is based on a fragmentary and weathered specimen; a phalanx IV-1 with a triangular proximal surface, and a dorsal facet narrower than the ventral one with a medial tilting is present also in Vespersaurus, Skorpiovenator and Aucasaurus. Although we regard Velocisaurus unicus as representative of a likely valid taxon, the discovery of additional specimens with new anatomical data will be essential for the taxonomy of this form.

\section{NEW ABELISAURID MATERIALS FROM THE ANACLETO FORMATION (CAMPANIAN, UPPER CRETACEOUS) OF PATAGONIA, ARGENTINA, SHED LIGHT ON THE DIAGNOSIS OF BRACHYROSTRA (THEROPODA, ABELISAURIDAE)}

\author{
M. A. Baiano ${ }^{1,2}$, R. A. Coria ${ }^{1}$, And J. I. CAnale 3 \\ ${ }^{1}$ CONICET-Museo Municipal “Carmen Funes”. Av. Córdoba 55, 8318 Plaza Huincul, Neuquén, Argentina. mbaiano@unrn.edu.ar \\ ${ }^{2}$ CONICET-IIPG-UNRN. Av. Roca 1242, 8332 General Roca, Río Negro, Argentina. \\ ${ }^{3}$ CONICET-Museo Paleontológico "Ernesto Bachmann". 8311 Villa El Chocón, Neuquén, Argentina.
}

Abelisauridae is a theropod clade with a wide distribution in the Upper Cretaceous of Gondwana. The best preserved abelisaurid record is represented by forms such as Skorpiovenator, Majungasaurus, Carnotaurus and Aucasaurus. The most distinguishable features of this family include ornamented snout bones and cranial roof; extremely reduced forelimbs; spherical humeral head; pickaxe-like lateral processes on caudal vertebrae; and axe-shaped cnemial crest. We report new abelisaurid materials found in the same quarry of a recently published abelisaurid specimen (MPCN-PV-69) including: a distal end of a unidentified neural spine; a mid-caudal vertebra; a distal part of a left metatarsal IV articulated with a proximal part of phalanx IV-1; and several phalanx of Digit IV. By reviewing that specimen and the holotypes of Skorpiovenator and Aucasaurus, we identified a new apomorphic feature that is shared with Brachyrostra, increasing the diagnosis of that clade. Abelisauroid features in MPCN-PV-69 include a triangular distal end of metatarsal IV; a phalanx IV1 with the proximal surface dorsoventrally taller than mediolaterally wide; a ventral surface transversely wider than the dorsal one, and the presence of medial tilting. Interestingly, certain features present in the phalanx IV-1 such as a ridge that spans from the proximodorsal projection and splits in two branches that surround an obliquely oriented and oval hyperextensor pit, is unquestionably present in all abelisaurids with a known Digit IV (except Majungasaurus), and not observed in any other theropod clade. Hence, here we propose the aforementioned combination of features as a possible synapomorphic character for the clade Brachyrostra. 


\title{
NEW ARCHOSAUR REMAINS FROM THE PORTEZUELO FORMATION (TURONIAN- CONIACIAN, UPPER CRETACEOUS) OF “LOS BASTOS" LOCALITY (SENILLOSA, NEUQUÉN PROVINCE, ARGENTINA)
}

\author{
F. Bellardini ${ }^{1}$ and M. A. Baiano 2,3 \\ 'Dirección Provincial de Patrimonio Cultural, Depto. de Paleontología. Vuelta De Obligado 50, 8300 Neuquén, Argentina. \\ flaviobellardini@gmail.com \\ ${ }^{2}$ CONICET-IIPG, Universidad Nacional de Río Negro. Roca 1242, 8332 Gral. Roca, Río Negro, Argentina \\ ${ }^{3}$ Museo Municipal "Carmen Funes". Av. Córdoba 54 (RN n²2), 8318 Plaza Huincul, Argentina.
}

In the central Neuquén Basin, the Portezuelo Formation is represented with extended fluvial outcrops, many of whom are well-known for yielded an abundant vertebrate fossil record. In this contribution we report new archosaur remains from "Los Bastos" locality (Neuquén Province, Patagonia, Argentina), which here are preliminary assigned to pterosaur, sauropod and theropod dinosaurs. Most of the fossils were found associated in situ in a well-cemented paleosoil composed by coarsegrained and browned sandstones, while other isolated materials were recovered few hundred meters away. Most relevant sauropod elements are: a fragmentary cone-chisel-like tooth with single high-angled wear-facet (MMS-PV-20), two procoelic anterior caudal vertebrae with neural arch beyond the anterior articular surface (MMS-PV-09-10), and a femur with anteroposteriorly well-compressed diaphysis and a prominent lateral bulge (MMS-PV-15). According to size and diagnostic features of each element we tentatively refer these bones to Titanosauria indet. Other materials are an incomplete theropod humerus (MMS-PV-18) with an internal tuberosity connected with humeral head and a low great tubercle, and four incomplete weakly-amphicoelous dorsal centra, with a shallow lateral fossa, which we assigned to Theropoda indet. (MMS-PV-22, 23, 24). An incomplete femur of a large pterosaur specimen (MMS-PV-21) with a wellmarked lesser trocanther and thin bone-wall was also found. In the "Los Bastos" locality, the Portezuelo Formation is associated to a meandering, high-sinuosity fluvial environment, as well as in type-locality. These new archosaur materials increase the Portezuelo Formation vertebrate fossil record for central Patagonia, and improve the faunal diversity regarded for Los Bastos locality of south Neuquén Province.

\section{CARACTERIZACIÓN SEDIMENTOLÓGICA DEL SITIO DE NUEVOS HALLAZGOS FOSILÍFEROS EN EL CAMPUS DE LA UNIVERSIDAD NACIONAL DEL COMAHUE - NEUQUÉN}

\footnotetext{
L. Berra ${ }^{1}$, M. Gasparini ${ }^{1}$, D. J. López ${ }^{1}$, M. Martínez ${ }^{1}$, N. Neme ${ }^{1}$, J. A. Paez ${ }^{1}$, S. Sánchez ${ }^{1}$ y C. Valderrama ${ }^{1}$

${ }^{1}$ Facultad de Ingeniería, Departamento Geología y Petróleo, Universidad Nacional del Comahue. Buenos Aires 1400, 8300 Neuquén, Patagonia, Argentina.berracinthyaliliana@gmail.com; martingasparini2008@hotmail.com; lopez.dario.j@outlook.com; maca.maca.maca@outlook.es; nasib.neme@gmail.com; julietapaezz@hotmail.com; santisancolo@gmail.com; ceci.valderrama@live.com

Los afloramientos del campus de la UNCo y zonas adyacentes han aportado numerosos restos fósiles, los cuales provienen casi en su totalidad de la Formación Bajo de la Carpa (Cretácico Superior), cuyo ambiente ha sido interpretado como depósitos de dunas e interdunas. Recientemente se produjo el hallazgo en la unidad suprayacente, correspondiente a la base de la Formación Anacleto (Cretácico Superior), de una pieza apendicular de un dinosaurio saurópodo (MUCPV-1558), constituyendo así el primer registro de vertebrado para esta unidad en este sector. El ejemplar fue hallado en depósitos de canales entrelazados efímeros. La sucesión analizada se compone por depósitos de canales y planicies de inundación o intercanal. Estos últimos son de carácter fangoso macizo con delgadas intercalaciones psamíticas interpretadas como depósitos de avenidas mantiformes. Los canales conforman un conjunto de cuerpos lateralmente amalgamados, confiriéndoles una estratoforma tabular, de gran extensión lateral y potencia menor al metro. Cada cuerpo canalizado individual se compone por depósitos psamíticos gruesos ocasionalmente sabulíticos, textural y composicionalmente inmaduros, dominados por depósitos de carácter tractivo (formas de lecho) integrados por las facies St, Se, Sh, y subordinadamente Sp. El desarrollo de sets individuales sumado a la escasa potencia de los cuerpos canalizados y la intercalación y/o interlaminación de fangolitas, permiten inferir el carácter efímero de estos canales. La morfología y el carácter de los depósitos sugieren un ambiente fluvial efímero en el área de estudio donde se encontró el ejemplar.
} 


\title{
LA FORMACIÓN BAJADA COLORADA Y SUS APORTES AL CONOCIMIENTO DE LOS VERTEBRADOS FÓSILES DEL CRETÁCICO TEMPRANO DE GONDWANA
}

\author{
J. I. Canale ${ }^{1,2}$, P. A. Gallina ${ }^{1,3}$, S. Apesteguía ${ }^{1,3}$, F. Riguett1 $1^{1,3}$, J. P. Garderes ${ }^{1,3}$ y A. Haluza ${ }^{2 *}$ \\ ${ }^{1}$ CONICET. \\ 2Área Laboratorio e Investigación, Museo Municipal "Ernesto Bachmann". Villa El Chocón, Neuquén, Argentina. juanignaciocanale@yahoo.com.ar \\ ${ }^{3}$ Área de Paleontología, Fundación de Historia Natural Félix de Azara, CEBBAD, Universidad Maimónides. Hidalgo 775, 1405 Ciudad Autónoma \\ de Buenos Aires, Argentina.pablo.gallina@fundacionazara.org.ar; sebapesteguia@gmail.com
}

Los afloramientos de la Formación Bajada Colorada (Berriasiano-Valanginiano), en su localidad tipo ubicada en el centrosur de la provincia de Neuquén, han sido objeto de exploraciones y excavaciones periódicas desde el año 2010, realizadas por los equipos del Museo "Ernesto Bachmann" de Villa El Chocón y la Fundación "Félix de Azara"-Universidad Maimónides de Buenos Aires. Como resultado, se han colectado numerosos y diversos restos de dinosaurios, destacándose osteodermos de posibles Tyreophora (Ankylosauria/Stegosauria?), nuevas especies de saurópodos Diplodocidae: Leinkupal laticauda Gallina et al., Dicraeosauridae: Bajadasaurus pronuspinax Gallina et al., y un saurópodo titanosaurio (MMCh-PV 228). Entre los dinosaurios terópodos se han colectado dientes aislados de megalosáuridos, un extremo proximal de tibia de un abelisáurido y el esqueleto parcial de un noasaurino. Una reciente colecta proveyó un extremo distal de tibia de abelisáurido (MMCh-PV 256), y una vértebra dorsal (MMCh-PV 257) y una tibia (MMCh-PV 258) asignables a Noasaurinae, incrementando el registro de estos taxones en esta formación. Esta asociación faunística muestra similitudes en su composición con las formaciones Lourinhã de Portugal y Tendaguru de Tanzania, del Jurásico Superior y Formación Kirkwood de Sudáfrica; del Cretácico Inferior. En el caso de Bajada Colorada coexisten algunos de los registros más modernos a nivel mundial de ciertos clados, como es el caso de diplodócidos y megalosáuridos, así como los más antiguos titanosaurios y noasaurinos. Teniendo en cuenta que una vasta cantidad de material se halla en proceso de preparación e incluso de extracción, nuestro conocimiento de esta fauna aumentará con seguridad considerablemente.

*Proyecto subsidiado por Municipalidad de Villa El Chocón, National Geographic Society (Grant \#W465-16) y Fundación Azara.

\section{PRESENCIA DE EXCAVACIONES DE VERTEBRADOS FÓSILES EN LA FORMACIÓN BAJO DE LA CARPA (SANTONIANO), PASO CÓRDOBA, RÍO NEGRO, ARGENTINA}

\author{
C. Cónsole-Gonella ${ }^{1}$, I. Díaz-Martínez ${ }^{*}$, S. De Valais², P. Citton ${ }^{2}$ y P. Paniceres $3 *$
}

${ }^{1}$ Instituto Superior de Correlación Geológica (INSUGEO), Universidad Nacional de Tucumán-CONICET. Miguel Lillo 205, 4000 San Miguel de Tucumán, Tucumán, Argentina.

${ }^{2}$ Instituto de Investigación en Paleobiología y Geología (IIPG), Universidad Nacional de Río Negro-CONICET. Av. Roca 1242, 8332 General Roca, Río Negro, Argentina.idiaz@unrn.edu.ar

${ }_{3}^{3}$ Municipalidad de General Roca. 8332 General Roca, Río Negro, Argentina.

La Formación Bajo de la Carpa (Grupo Neuquén) aflora ampliamente en el borde de la margen sur del Río Negro, en el Área Municipal Natural Protegida Paso Córdoba, provincia de Río Negro, Argentina. En dichos afloramientos son abundantes los restos óseos fósiles de serpientes, cocodrilos y dinosaurios terópodos no avianos. En la presente unidad, predominan las areniscas de grano medio a grueso, mal seleccionadas, que han sido relacionadas con un ambiente fluvial de moderada a alta energía. También se han identificado varios niveles de paleosuelos, los que indicarían periodos de cierta estabilidad del sustrato para su edafización. Relacionados a estos niveles, se han encontrado estructuras tubulares de orientación predominantemente vertical, perpendiculares a la estratificación en areniscas de grano medio-grueso. Las mismas poseen un largo máximo observado de $40 \mathrm{~cm}$ y un diámetro promedio de 10 a $12 \mathrm{~cm}$, además exhiben constricciones y ensanchamientos a lo largo de todo su curso. La geometría de estas estructuras en algunos casos conforma patrones débiles en $L$, con una tortuosidad baja (1-2). En una de estas, se ha observado un ensanchamiento elipsoidal, con el eje mayor paralelo a la estratificación. Estas estructuras poseen un relleno masivo, de litología diferente al de la roca hospedante, que consiste en una arenisca de grano más fino y que presenta colores en tonos más claros debido a oxidación diferencial. Estas estructuras son interpretadas como excavaciones y se asignan provisoriamente a galerías de morada y/o reproducción de pequeños vertebrados durante los momentos de estabilización del sistema fluvial.

*Proyecto PI UNRN 40-A-580 a L. Salgado. 
PE-APA 20 (2) - Libro de RESÚMENES

\title{
ESTUDIO PRELIMINAR DE LAS PATOLOGÍAS ÓSEAS DE BONAPARTESAURUS RIONEGRENSIS (DINOSAURIA: HADROSAURIDAE)
}

\author{
P. Cruzado-Caballero ${ }^{1}$, A. Lecuona ${ }^{1}$, I. A. Cerda ${ }^{1,2}$ e I. Díaz-Martínez ${ }^{1 *}$
}

${ }^{1}$ CONICET, Instituto de Investigación en Paleobiología y Geología (IIPG-UNRN). Av. Roca 1242, 8332 General Roca, Río Negro, Argentina. pccaballero@unrn.edu.ar; alecuona@unrn.edu.ar; nachocerda6@yahoo.com.ar; idiaz@unrn.edu.ar

2Museo Provincial "Carlos Ameghino". Belgrano 1700, Paraje Pichi Ruca (predio Marabunta), 8300 Cipolletti, Río Negro, Argentina.

Las paleopatologías encontradas en organismos fósiles proporcionan valiosa información sobre aspectos paleobiológicos y paleoecológicos. El hadrosaurino Bonapartesaurus rionegrensis Cruzado-Caballero y Powell (MPCA-PvSM2) de la Formación Allen (Cretácico Superior, Río Negro), registra las primeras patologías óseas de este clado en Gondwana. Las patologías se localizan en varias espinas neurales caudales y en el metatarsiano II del pie izquierdo. Estos huesos presentan un notable sobrecrecimiento del tejido óseo debido a las fracturas de estos. Las patologías de las espinas neurales se localizan a media altura y algunas de ellas presentan una marcada desviación lateral. El tejido patológico del metatarsiano ocupa casi toda la longitud de este, expandiéndose lateralmente hasta el límite de las caras articulares. Una tomografía realizada al pie reveló que la fractura fue desplazada y posteriormente curada. A nivel histológico, el sobrecrecimiento del metatarsiano exhibe un alto grado de porosidad, el cual contrasta con el aspecto compacto del tejido subyacente (hueso no patológico). El hueso patológico primario es de tipo entretejido, exhibiendo una gran cantidad de espacios de reabsorción, muchos de ellos revestidos por tejido lamelar secundario. Esto último sugiere de un activo proceso de recambio del tejido patológico. Las fracturas presentan un callo bien formado lo que indica una completa reparación y que los eventos traumáticos que las produjeron no fueron los causantes de su muerte. Las patologías observadas pudieron haber afectado el comportamiento de Bonapartesaurus (e.g., locomoción), hipótesis que deberá ser puesta a prueba con análisis funcionales y anatómicos de los miembros posteriores y cintura pélvica.

*Proyecto subsidiado por beca jóvenes investigadores APA 2016 (PC-C), PI UNRN 40-A-737 (PC-C, A.L.) y PICT $2016-0491$ (PC-C).

\section{CORRELACIÓN ESTRATIGRÁFICA DE NUEVOS HALLAZGOS PALEONTOLÓGICOS EN LA LOCALIDAD TIPO DE LA FORMACIÓN CERRO LISANDRO, CRETÁCICO SUPERIOR, CUENCA NEUQUINA (SENILLOSA, PATAGONIA, ARGENTINA)}

\author{
F. DIAZ ${ }^{1,2}$ Y F. BELLARDINI2,3
}

\author{
1Universidad Nacional del Comahue, Facultad de Ingeniería. Buenos Aires 1400, 8300 Neuquén, Neuquén, Argentina.fausto_di@hotmail.com.ar \\ ${ }^{2}$ Museo Municipal de Ciencias Naturales. Av. Olascoaga s/n, 8320 Senillosa, Neuquén, Argentina. \\ ${ }^{3}$ Dirección Provincial de Patrimonio Cultural, Depto. de Paleontología. Vuelta de Obligado 50, 8300 Neuquén, Argentina. \\ flaviobellardini@gmail.com
}

La Formación Cerro Lisandro representa un paquete de sedimentitas continentales de ambiente fluvial caracterizado por una alternancia de pelitas y psamitas de edad Turoniana. El registro fósil para esta unidad es escaso, limitado a materiales aislados y fragmentarios, compuestos por dientes de terópodos, placas de caparazones de tortugas, bivalvos de agua dulce y elementos referibles a dinosaurios indet. En este trabajo presentamos nuevos materiales fósiles procedentes de la localidad tipo y la correlación estratigráfica de tres nuevos sitios fosilíferos con sus implicancias paleoambientales. En el sitio $n^{\circ} 1$, del cual procede un diente casi completo de dinosaurio terópodo (MMS-PV-12), aflora un paquete de areniscas medias, de color grisáceo, escasamente consolidadas, con estructuras en artesa y una acreción lateral bien definida. En el sitio $n^{\circ} 2$, ubicado unos 400 m más al noroeste, en un nivel de areniscas grisáceas, poco consolidadas proceden fragmentos de caparazones de tortugas (MMS-PV-29) y elementos de dinosaurios indet. (MMMS-PV-30), mientras que en el paquete de fangolitas rojizas subyacentes se hallaron porciones de valvas desarticuladas de bivalvos referibles a Diplodon sp. (MMSPI-21) (Sitio $n^{\circ} 3$ ). La correlación de los perfiles estratigráficos en estos sitios indica una alternancia de facies pelíticas y psamíticas, en las cuales se registran por lo menos tres niveles fosilíferos, dos referibles a depósitos de canales de tipo meandroso (artesas y acreción lateral definida) y otro (sitio $n^{\circ} 3$ ) a una planicie fangosa de inundación. Estos nuevos hallazgos en estos sitios permitirán correlacionar a nivel regional futuros hallazgos en la Formación Cerro Lisandro dentro de la Cuenca Neuquina. 
POSSIBLE POSTCRANIAL REMAINS OF PEHUENCHESUCHUS ENDERI TURNER AND CALVO, 2007 (CROCODYLIFORMES, SEBECIDAE?)

\author{
L. S. FILIPPI ${ }^{1}$ AND R. D. JUÁREZ VALIERI ${ }^{2,3}$ \\ ${ }^{1}$ Museo Municipal Argentino Urquiza. Chos Malal s/n., 8319 Rincón de los Sauces, Neuquén, Argentina. Isfilippi@gmail.com \\ ${ }^{2}$ Secretaría de Cultura de la Provincia de Río Negro, Museo Provincial Carlos Ameghino. Belgrano 2150, 8324 Cipolletti, Río Negro, Argentina. \\ rubendjuarez@gmail.com \\ ${ }^{3}$ Museo de Ciencias de Añelo. Calle 6 S/N, 8305 Añelo, Neuquén, Argentina.
}

The revision of cranial material of the titanosaurian dinosaur Rinconsaurus caudamirus Calvo and González Riga, coming from levels actually regarded belonging to the Bajo de la Carpa Formation (Neuquén Group), resulted in the reinterpretation the prefrontal bone (MAU-Pv-CRS-102, paratype) as belonging to a crocodyliform right ilium. This assignment is based on a series of anatomical features present in the material that allows it to be compared with various subgroups within Crocodyliformes, and particularly within Ziphosuchia. The presence of a short preacetabular process, characteristic of Mesoeucrocodylia. The dorsoventral development of the same differentiates it from Neosuchia. The postacetabular process is directed horizontally, unlike the neosuchians and Baurusuchus albertoi, and its proximodistal extension is similar to that present in the Uruguaysuchus aznarezi, Araripesuchus tsangatsangana and Mahajangasuchus insignis, but shorter than in Sebecus icaeorhinus. The development of the supracetabular crest is subhorizontal as in ziphosuchians, although not reaching the degree of development of Notosuchus terrestris and Baurusuchus albertoi, corresponding to that present in both uruguaysuchids and Sebecia. Contact with two sacral vertebrae is verified in the specimen, as in all ziphosuchians except baurusuchids and Mariliasuchus amarali, which presents three articular facets. The relative size and the morphology coinciding with that of the sebecians allows to infer that this ilium represents additional material of Pehuenchesuchus enderi Turner and Calvo, which was found in the same quarry. This possibility implies access to novel postcranial information that can help clarify the phylogenetic relationships of this taxon.

\title{
FEEDING TRACES ON SAUROPOD DORSAL VERTEBRAE FROM UPPER CRETACEOUS (BAJO DE LA CARPA FORMATION, SANTONIAN) OF NORTH OF NEUQUÉN PROVINCE
}

\author{
L. S. FILIPPI ${ }^{1}$ AND F. BELLARDINII'3
}

\author{
${ }^{1}$ Museo Municipal Argentino Urquiza. Chos Malal s/n., 8319 Rincón de los Sauces, Neuquén, Argentina. Isfilippi@gmail.com \\ 2Dirección Provincial de Patrimonio Cultural, Departamento de Paleontología. Vuelta De Obligado 50, 8300 Neuquén, Argentina. \\ flaviobellardini@gmail.com \\ ${ }^{3}$ Museo Municipal de Ciencias Naturales. Av. Olascoaga s/n, 8320 Senillosa, Neuquén, Argentina.
}

In non-avian dinosaur fossil record, bite marks are not common or mostly ambiguous or of uncertain origin, however they can represent the better direct evidences of interactions between carnivorous and their prey. In this contribution, we present the evidence of feeding traces on an isolated sauropod element from Cerro Overo, Bajo de la Carpa Formation outcrops (Upper Cretaceous, Santonian) of north of Neuquén Province (Patagonia, Argentina). The specimen represents a partial sauropod dorsal vertebral centrum (MAU-Pv-CO-651). In lateroventral surface of the centrum three types of marks are preserved. They include a first series of three large and deep longitudinal grooves, perpendicularly oriented respect to the antero-posterior axis of centrum and partially filled with matrix, a second series of three shallower and smaller longitudinal grooves and, finally, a series of small pits that we tentatively regarded as perforations. These kinds of marks can be regarded as feeding traces produced by macro-vertebrates, probably during scavenging. However, we consider both series of three longitudinal grooves as "drag marks" and could be referred to the bite-marks of theropod dinosaurs. Furthermore, the small perforations could be representing "punctures", and represent the product of bite-marks of crocodiles. In relation to potential carnivorous in the fossil record for the area, we can report: the abelisaur Viavenator exxoni Filippi et al. and the peirosaur Kinesuchus overoi Filippi et al. These evidences suggest that the bite-marks on this material may be the product of the interaction of several scavengers as it happens in current ecosystems. 


\title{
RELACIONES ESTRATIGRÁFICAS Y PALEOAMBIENTE DE LOS DEPÓSITOS PORTADORES DE LOS RESTOS DE ARGENTINOSAURUS HUINCULENSIS BONAPARTE Y CORIA, 1993 Y MAPUSAURUS ROSEAE CORIA Y CURRIE, 2006, CRETÁCICO SUPERIOR DE LA CUENCA NEUQUINA
}

\author{
A. C. GARRIDO $0^{1,2}$ \\ ${ }^{1}$ Museo Provincial de Ciencias Naturales "Prof. Dr. Juan A. Olsacher", Dirección Provincial de Minería. Etcheluz y Ejército Argentino, 8340 \\ Zapala, Neuquén, Argentina. albertocarlosgarrido@gmail.com \\ ${ }^{2}$ Centro de Investigación en Geociencias de la Patagonia, Departamento Geología y Petróleo, Facultad de Ingeniería, Universidad Nacional del \\ Comahue. Buenos Aires 1400, 8300 Neuquén, Argentina.
}

El Argentinosaurus huinculensis y el Mapusaurus roseae constituyen dos dinosaurios emblemáticos de la Cuenca Neuquina. Reconocidos por su gran tamaño, se ubican respectivamente entre los dinosaurios herbívoros y carnívoros más grandes del mundo. Si bien es conocida su proveniencia de la Formación Huincul (Grupo Neuquén, Cretácico Superior), su ubicación precisa dentro de la columna sedimentaria es hasta el momento incierta. La Formación Huincul presenta una sección inferior dominada por un sistema fluvial de alta sinuosidad y carga fangosa a mixta, y una sección superior caracterizada por el desarrollo de depósitos fluviales que gradan regionalmente desde sistemas entrelazados gravo-arenosos a sinuosos de carga arenosa. Mientras la sección inferior (70 m de espesor máximo) indicaría condiciones climáticas cálidas y semiáridas dominantes, la sección superior (195 m de espesor máximo) denota el desarrollo de una estacionalidad más marcada, con periodos de mayor humedad relativa. Perfiles estratigráficos realizados en los sitios de hallazgo, y localidades intermedias, permiten establecer que $A$. huinculensis y $M$. roseae se ubican en el tramo medio de la sección superior, mediando entre ellos una distancia relativa en la vertical del orden de los $30 \mathrm{~m}$. En ambos casos, los restos aparecieron asociados a depósitos de canales gravo-arenosos bajo la forma de concentraciones residuales de fondo de canal. El gran tamaño de los elementos óseos imposibilitó su transporte a grandes distancias y su mayor dispersión, constituyendo sus yacimientos concentraciones de tipo parautóctonas.

\section{RECUPERO, CHEQUEO, RESTAURACIÓN Y REORGANIZACIÓN DE LAS COLECCIONES DEL MUSEO PROVINCIAL DE CIENCIAS NATURALES "PROF. DR. JUAN A. OLSACHER" (MOZ). ZAPALA, PROVINCIA DEL NEUQUÉN}

\author{
A. C. Garrido ${ }^{1,2}$, B. Bollini ${ }^{1}$ y C. Musso PeruláN ${ }^{1,3}$
}

${ }^{1}$ Museo Provincial de Ciencias Naturales "Prof. Dr. Juan A. Olsacher", Dirección Provincial de Minería. Etcheluz y Ejército Argentino, 8340 Zapala, Neuquén, Argentina.mozapala@yahoo.com.ar

${ }^{2}$ Centro de Investigación en Geociencias de la Patagonia, Departamento Geología y Petróleo, Facultad de Ingeniería, Universidad Nacional del Comahue. Buenos Aires 1400, 8300 Neuquén, Argentina.

${ }^{3}$ Asentamiento Universitario Zapala (AUZa), Facultad de Ingeniería, Universidad Nacional del Comahue. Av. 12 de Julio y Rahue, 8400 Zapala, Neuquén, Argentina.

A inicios del año 2008 el MOZ comenzó una etapa de reorganización institucional que incluyó, entre otros, la modernización de sus instalaciones y la adecuación de su estructura organizacional; creándose un reglamento administrativo que regula y direcciona el funcionamiento de cada área y/o departamento de la institución. Las colecciones del MOZ constituyeron desde un primer momento el principal foco de atención y preocupación, cuyo estado de situación fue señalado en aquel momento como "crítico". Aproximadamente el 85\% de las piezas de colección se encontraban fuera de la institución, distribuidas en diversos predios de la ciudad de Zapala. Además, varias centenas de piezas se encontraban en calidad de préstamo en diversas instituciones del país, algunas de las cuales revestían más de dos décadas y media en dicha situación. Las tareas de reorganización contemplaron el traslado de las colecciones a las instalaciones del MOZ, chequeo de las piezas existentes, restauración de las piezas que se presentaban deterioradas, y el recupero de los materiales en préstamo. Al 2019, los resultados son los siguientes: 96 Tn de materiales fueron ubicados y trasladados al MOZ, 950 piezas paleontológicas fueron restauradas, 1.014 piezas en préstamo fueron recuperadas y se prepararon y acondicionaron 670 nuevas piezas de colección. Por otro lado, los trabajos de campo han permitido incrementar desde el año 2008 a la fecha en aproximadamente 2 veces y media el número de piezas paleontológicas. Actualmente el MOZ posee en sus registros un ingreso de 15.341 piezas paleontológicas, 6.782 piezas minerales y 750 piezas litológicas. 


\title{
ANÁLISIS DE FACIES Y MICROFACIES SEDIMENTARIAS DE LOS NIVELES RICOS EN INVERTEBRADOS DEL CRETÁCICO SUPERIOR DE PASO CÓRDOBA, GENERAL ROCA, RÍO NEGRO
}

\author{
A. Gigli1 ${ }^{1}$ I. Díaz Martínez ${ }^{1}$ y F. M. Archuby²
}

${ }^{1}$ Instituto de Investigación en Paleobiología y Geología (IIPG), CONICET y UNRN. Av. Roca 1242, 8332 General Roca, Río Negro, Argentina. ayegeogi@gmail.com; inaportu@hotmail.com

${ }^{2}$ Centro de Estudios Integrales de la Dinámica Exógena (CEIDE, UNLP) y CONICET. Diagonal 113 nro. 469, 1900 La Plata, Buenos Aires, Argentina.farchuby@gmail.com

El área Natural Municipal Protegida Paso Córdoba se encuentra al sur de la localidad de General Roca, Río Negro. En esta zona afloran las fangolitas fluvio-lacustres de la Formación Anacleto (Campaniano inferior) y las areniscas eólicas de la Formación Allen (Campaniano superior-Maastrichtiano superior). Se analizaron cinco muestras rocosas tomadas de distintos niveles tabulares de las formaciones Anacleto, Allen y de la transición entre ambas. Se estudió un nivel silicificado con una representación autóctona de la comunidad. Se identificaron dos tipos de facies sedimentarias: 1- Facies de grano grueso (rudstones arenosos y areniscas aloquímicas), 2- Facies de grano fino (mudstones y wackestones bioclásticos). El tipo 1 está representado por rocas mixtas con dominancia de bioclastos (mayoritariamente bivalvos mayores a $10 \mathrm{~mm}$ y algunos ostrácodos) y granos de arena, con cemento esparítico. Las valvas se presentan recristalizadas a modo de cristales de calcita esparítica en forma de mosaico con crecimiento desde los bordes hacia el centro y rodeados por micrita. Los bioclastos se encuentran densamente empaquetados, desarticulados y dispuestos convexos (mayormente) y cóncavos hacia arriba, normalmente completos (no fracturados). Las facies del tipo 2 son de grano fino y están conformadas principalmente por micrita (microesparita), con oogonios de carofitas y valvas desarticuladas de ostrácodos de entre 0,5 y $1 \mathrm{~mm}$, recristalizados a calcita, con tendencia a disponerse convexas hacia arriba. Se interpreta que los tipos de facies 1 y 2 representan relictos de la comunidad hallada en el nivel silicificado (i.e., autóctono) en condiciones de alta y baja energía respectivamente.

\section{MADURACIÓN SEXUAL EN MUYELENSAURUS PECHENI (SAUROPODA, TITANOSAURIA) INFERIDA A PARTIR DE LA HISTOLOGÍA DE HUESOS APENDICULARES}

\author{
R. Gonzálezz', I. A. Cerrda², J. O. Calvo ${ }^{3}$ y B. J. González Riga ${ }^{4 *}$ \\ ${ }^{1}$ Centro de Ecología Aplicada del Litoral (CECOAL). Ruta 5, Km 2,5, 3400 Corrientes, Argentina. romigonzl95@gmail.com \\ ${ }^{2}$ Instituto de Investigaciones en Paleobiología y Geología, Universidad Nacional de Río Negro-CONICET. Museo Provincial Carlos Ameghino, \\ Belgrano 1700, Paraje Pichi Ruca (predio Marabunta), 8300 Cipolletti, Río Negro, Argentina. nachocerda6@gmail.com \\ 3universidad Nacional del Comahue, Centro Paleontológico Lago Barreales, Proyecto Dino. Neuquén, Argentina. jocalvo40@yahoo.com.ar \\ ${ }^{4}$ Laboratorio de Dinosaurios, Facultad de Ciencias Exactas y Naturales, Universidad Nacional de Cuyo, CONICET. Avenida Padre Contreras \\ 1300, Edificio ECT, Parque General San Martín, 5500 Mendoza, Argentina.bgonzalez@fcen.uncu.edu.ar
}

Muyelensaurus pecheni Calvo, Gonzalez-Riga y Porfiri es un titanosaurio procedente de la Formación Plottier (Cretácico Superior), aflorante en cercanías de Rincón de los Sauces, Neuquén. Se han asignado a esta especie varios individuos de diferentes estadios ontogenéticos. Aquí se propone estudiar el grado de correlación temporal entre la maduración sexual (reflejada en su osteohistología) y la maduración esqueletal (reflejada en el grado de fusión de las suturas neurocentrales). Se analizó la microestructura ósea de dos fémures correspondientes a un individuo adulto (MRS-Pv-89) y un juvenil (MRSPv-429), y dos húmeros de individuos adultos (MRS-Pv-70, MRS-Pv-352) referidos a Muyelensaurus y cuyo estadio ontogenético fue inferido a priori según la longitud de los huesos estilopodiales. Ambos fémures están conformados principalmente por un tejido fibrolamelar con vascularización longitudinal y circunferencial. Por su parte ambos humeros, se caracterizaron por presentar un tejido principalmente secundario con una delgada capa de tejido primario en la corteza externa. Las características detectadas son similares a las reportadas en macronarios basales y diplodocoideos asignados a individuos subadultos (MRS-Pv-429) y adultos (MRS-Pv-89/70/352). Todos los elementos exhiben en la corteza externa marcas de crecimiento (líneas de crecimiento detenido y annuli). La observación de una disminución en el espaciado entre marcas sucesivas en MRS-Pv-89/70 y MRS-Pv-352 sugiere que dichos individuos habrían alcanzado la madurez sexual, y que este rasgo precede a la maduración esqueletal, evidenciado por la completa fusión de las suturas neurocentrales de todas las vértebras.

*Proyecto subsidiado por PICT 2015-1021. 


\title{
DINOSAURIOS COMO HERRAMIENTA PARA EL DESARROLLO REGIONAL: CASO EJEMPLO MUESTRA “DINOSAURIOS Y OTRAS BESTIAS PREHISTÓRICAS DE RÍO NEGRO”
}

\author{
R. D. Juárez Valieri ${ }^{1,2}$, J. E. Aravena Melo ${ }^{1}$, J. C. Muñoz ${ }^{1}$, R. Fasano ${ }^{1}$ y A. W. J. Laurín ${ }^{1}$
}

1Secretaría de Cultura de la Provincia de Río Negro, Museo Provincial Carlos Ameghino. Belgrano 2150, 8324 Cipolletti, Río Negro, Argentina. rubendjuarez@gmail.com

${ }^{2}$ Museo de Ciencias de Añelo. Calle 6 S/N, 8305 Añelo, Neuquén, Argentina.

Durante el período 2017-2019, el Museo Provincial Carlos Ameghino (MPCA) de la ciudad de Cipolletti dependiente de la Secretaría de Cultura de la Provincia de Río Negro, ha llevado un proyecto de difusión mediante una muestra itinerante denominada "Dinosaurios y otras bestias prehistóricas de Río Negro". El mismo presenta a la vez una arista educativa, permitiendo acceder a las instituciones de los diferentes niveles de enseñanza, en particular aquellos de localidades donde se cuenta con poco o nulo acceso a museos u otras instituciones donde los estudiantes puedan aprender y apreciar aspectos relativos a paleontología y el patrimonio en general. Sirve a la vez como una campaña de concientización sobre la valoración y protección del patrimonio paleontológico, brindando a las localidades asesoramiento sobre aspectos referentes a procedimientos tendientes a cubrir denuncias de hallazgos y brindar una comprensión general sobre el potencial paleontológico de sus áreas de influencia. Como resultado se recabaron múltiples denuncias de hallazgos. Finalmente, el otro aspecto cubierto en el proyecto es el de insertar la muestra como un elemento más de atracción turística de la provincia, realizándose durante temporada de afluencia turística a destinos vacacionales, aportando un atractivo regional adicional. Durante el periodo transcurrido se han visitado 24 localidades dentro de la provincia y 1 en otra jurisdicción. En base a estimaciones realizadas en conjunto con los municipios y comisiones de fomento donde fue presentado se ha contado con la afluencia de más de ciento ochenta cinco mil visitantes.

\section{A NEW PHYLOGENETIC PERSPECTIVE ON THE CRANIAL VARIABILITY OF THE TITANOSAURIAN SAUROPODS}

\author{
R. D. JuÁrez Valieri ${ }^{1,2}$, L. S. FILIPPI ${ }^{3}$, AND S. D. Ríos ${ }^{4}$
}

1Secretaría de Cultura de la Provincia de Río Negro, Museo Provincial Carlos Ameghino. Belgrano 2150, 8324 Cipolletti, Río Negro, Argentina. rubendjuarez@gmail.com

${ }^{2}$ Museo de Ciencias de Añelo. Calle 6 S/N, 8305 Añelo, Neuquén, Argentina.

${ }_{3}^{3}$ Museo Municipal Argentino Urquiza. Chos Malal s/n., 8319 Rincón de los Sauces, Neuquén, Argentina. Isfilippi@gmail.com

4Departamento de Arqueología y Paleontología, Secretaría Nacional de Cultura. Asunción, Paraguay. sergiord40@gmail.com

Titanosauria is a remarkable clade of late Mesozoic and globally distributed sauropod dinosaurs with dozens of referred species. However, craniofacial knowledge is restricted to a few taxa, with only five taxa known from nearly complete skulls, and three of them contain little or nule postcranial information. Due to this, most previous studies related to titanosaur phylogeny do not include many of these taxa. Additionally, the craniomandibular morphological characters included in earlier analyses are intended to differentiate titanosaurs from other sauropods clades but do not represent intragroup variations. This has resulted in the underestimation of cranial morphological diversity and its distribution within Titanosauria. Here we present a new phylogenetic analysis from a new data set derived from Carballido and colleagues of 576 morphological characters, including 276 craniomandibular and dental characters. 47 new characters have been incorporated and 62 previously utilized have been modified to incorporate the variation among titanosaurs. The taxon sampling includes 98 taxa of sauropods, including 47 supposed titanosaur taxa (26 of these preserving craniomandibular and dental information). The results recover to Sarmientosaurus outside Titanosauria and Choconsaurus as an Epachthosaurinae. Nemegtosauridae is an early branch of titanosaurs sister to Tapuiasaurus and more derived taxa (mdt). Lithostrotia includes an early branch of monophyletic clade Rapetosaurus plus Malawisaurus and are sister taxa to Jainosaurus and mdt. Saltasaurines and their close relatives are the sister clade to Colossosauria. Among the later, the lognkosaurians and Narambuenatitan are successive sister taxa to aeolosaurines and rinconsaurians, which includes the Antarctosaurus-like highly derived forms. 


\title{
PEQUEÑOS ANIMALES EN TIEMPOS DE GIGANTES: TÉCNICAS APLICADAS A LA RECUPERACIÓN DE PEQUEÑ̃OS VERTEBRADOS FÓSILES PROVENIENTES DE LA FORMACIÓN CANDELEROS (CENOMANIANO-TURONIANO), CERRO POLICÍA, RÍO NEGRO, ARGENTINA
}

\author{
J. Kaluza ${ }^{1,3}$, P. Varela ${ }^{2}$ y S. Apesteguia ${ }^{1}$ \\ ${ }^{1}$ CONICET-Fundación Félix de Azara, Universidad Maimónides. Hidalgo 775, 1405 Ciudad Autónoma de Buenos Aires, Argentina. \\ yojonatan@hotmail.com; sebapesteguia@gmail.com \\ ${ }^{2}$ Instituto de Estudios Andinos "Don Pablo Groeber", IDEAN-UBA- CONICET. Int. Güiraldes 2160, Ciudad Universitaria, Ciudad Autónoma de \\ Buenos Aires, Argentina.pdvarela@gl.fcen.uba.ar \\ ${ }^{3}$ Red Paleontológica U-Chile, Laboratorio de Ontogenia y Filogenia, Departamento de Biología, Facultad de Ciencias, Universidad de Chile. \\ Av. Las Palmeras 3425, Santiago, Chile.
}

Al noroeste de la Planicie de Rentería, al sur del embalse Ezequiel Ramos-Mexía (provincia de Río Negro), afloran, a lo largo de una franja de 50 km, depósitos de la Formación Candeleros, una unidad litoestratigráfica del Cretácico Superior y más de 95 millones de años de antigüedad. La localidad ha sido foco de estudio desde fines de los años ' 90 y es portadora de una gran diversidad faunística. Preserva característicamente vertebrados fósiles de mediano a pequeño tamaño, entre los que se destacan pequeños terópodos, cocodrilos terrestres, esfenodontes, serpientes basales con extremidades, lagartos y mamíferos driolestoideos. Si bien algunos de los hallazgos muestran evidencias claras de exposición a los agentes de meteorización y carroñeros, los esqueletos en general son de preservación excepcional, debido al rápido enterramiento sufrido por las grandes dunas de arena del antiguo desierto de Kokorkom. En febrero del 2014, un equipo multidisciplinario conformado por profesionales de distintas instituciones, realizó trabajos de extracción de vertebrados fósiles a unos $30 \mathrm{~km}$ noroeste del pueblo de Cerro Policía. El presente trabajo expone brevemente las técnicas de extracción y preparación mecánica aplicadas a vertebrados de pequeño tamaño. La extracción de bloques por cortadora de roca, la elaboración de bochones, la preparación mecánica por medio de martillos neumáticos, así como la utilización de técnicas no invasivas como la iluminación ultravioleta en el análisis y preparación de piezas fósiles, son algunos de los procedimientos aquí expuestos.

\section{HACIA UNA RECONSTRUCCIÓN DE LOS ECOSISTEMAS TERRESTRES DEL CRETÁCICO LA CUENCA NEUQUINA}

\author{
L. C. A. Martínez 1,2 , J. Porfiri ${ }^{3}$ y A. C. Garrido4,5 \\ ${ }^{1}$ Instituto de Botánica Darwinion (ANCEFN - CONICET). Labardén 200, CC22,1642 San Isidro, Buenos Aires, Argentina. gesaghi@gmail.com \\ 2Cátedra Paleobotánica, Facultad de Ciencias Naturales y Museo, Universidad Nacional de La Plata. Avenida 122 y 60, 1900 La Plata, Argentina. \\ ${ }^{3}$ Museo de Ciencias Naturales, Universidad Nacional del Comahue. Buenos Aires 1400, 8300 Neuquén, Argentina. \\ juan.porfiri@central.uncoma.edu.ar \\ ${ }^{4}$ Museo Provincial de Ciencias Naturales "Prof. Dr. Juan A. Olsacher", Dirección Provincial de Minería. Etcheluz y Ejército Argentino, 8340 \\ Zapala, Neuquén, Argentina. albertocarlosgarrido@gmail.com \\ ${ }^{5}$ Centro de Investigación en Geociencias de la Patagonia, Departamento Geología y Petróleo, Facultad de Ingeniería, Universidad Nacional \\ del Comahue. Buenos Aires 1400, 8300 Neuquén, Argentina.
}

Por sus características intrínsecas, las plantas son excelentes indicadoras de las condiciones del medio en el que habitan. De hecho, cualquier cambio ambiental repercute directamente en la flora y, por ende, influye fuertemente en el resto de la biota acompañante. Teniendo en cuenta esta premisa, se definen asociaciones sobre la base del registro de las plantas fósiles de determinadas unidades cretácicas de la Cuenca Neuquina (formaciones Bajada Colorada, Huincul y Allen). Se estudiaron los anillos de crecimiento y se aplicaron diversos índices (vulnerabilidad, mesomorfía, pariente próximo, diversidad, etc.), para así inferir las condiciones paleoambientales bajo las cuales se desarrollaron las sucesiones sedimentarias en estudio y evaluar la productividad de los ecosistemas cretácicos. En este sentido, se observa en los depósitos cretácicos de la Cuenca Neuquina una variación en las composiciones florísticas, lo que estaría relacionado con cambios ambientales ocurridos a lo largo de este período. En la Formación Bajada Colorada, las comunidades vegetales están formadas por bosques con un régimen templado-cálido y estacional; los que se contrastan con los bosques cálidos, húmedos y no estacionales de la Formación Huincul. Por su parte, la vegetación registrada en la Formación Allen, también se distingue por haberse desarrollado en un ambiente cálido, estacional y con un menor régimen hídrico respecto a las anteriores. Dichas comunidades vegetales se cotejaron con la paleofauna de vertebrados registrada en cada formación, pudiéndose de esta manera relacionar los taxones de vertebrados de cada unidad con el ambiente inferido por medio del estudio de las plantas fósiles. 


\title{
TAIL ANATOMY OF ALVAREZSAUROIDS (THEROPODA, COELUROSAURIA)
}

\author{
J. G. Meso ${ }^{1}$, J. I. Canale ${ }^{1,2}$, and L. Salgado ${ }^{1 *}$ \\ ${ }^{1}$ CONICET-IIPG, Universidad Nacional de Río Negro. Av. Roca 1242, 8332 Gral. Roca, Río Negro, Argentina. jgmeso@unrn.edu.ar \\ 2Museo Paleontológico "Ernesto Bachmann". 8311 Villa El Chocón, Neuquén, Argentina.
}

The anatomy of the alvarezsauroid tail has been poorly described in the literature, although it shows a peculiar combination of characters that make it unique among theropods. To this respect, the most complete and informative alvarezsauroid taxa are Alvarezsaurus calvoi Bonaparte, Shuvuuia deserti Chiappe et al., Haplocheirus sollers Choiniere et al., and Linhenykus monodactylus Xu et al., 2011. The alvarezsauroid tail is characterized for being the longest among coelurosaurs in both number of vertebrae and length. The vertebrae have procoelous and mediolaterally compressed centra, subtriangular and laterodistally directed transverse processes, short pre and postzygapophyses, and neural spines low, posteriorly inclined, and taller than wide in lateral view. The most proximal centra are ventrally sharp. These osteological traits, in adittion to the particular morphology of the intervertebral joint and inferred musculature, suggest a specific function of the tail, with an exceptional capacity of rotational inertia, and probably a marked development of M. spinalis and M. caudofemoralis, comparable to some extent with extant anteaters and pangolins. This provides for the first time new details of the tail anatomy and function among alvarezsaurian coelurosaurs. The preliminary results of this study support previous ideas, based on the morphology of the alvarezsaurid forelimb and skull, which suggest that this group occupied an ecological niche equivalent to that of anteaters or pangolins. Nevertheless, this hypothesis should be tested further under more complex biomechanical analyses.

*Proyecto subsidiado por PI-UNRN 40-A-580.

\section{RECONSTRUCCIÓN PALEOGEOGRÁFICA DE LA FORMACIÓN ALLEN (CAMPANIANO SUPERIOR- MAASTRICHTIANO INFERIOR) DEL GRUPO MALARGÜE Y SU RELACIÓN CON LAS FAUNAS EN EL SECTOR ORIENTAL DE LA CUENCA NEUQUINA}

\section{R. A. ORTIZ1}

1Rhode 5220, General Roca, 8332 Río Negro, Argentina.paleoroca@yahoo.com.ar

Se presenta la reconstrucción paleogeográfica de la Formación Allen (Grupo Malargüe, Campaniano superior-Maastrichtiano inferior) en el sector oriental de la Cuenca Neuquina, basado en el análisis sedimentológico, estratigráfico y contenido paleontológico de tres localidades: Paso Córdoba, Cinco Saltos y Gómez, en el centro-norte de la provincia de Río Negro. En Paso Córdoba el miembro inferior de la Formación Allen está representado por canales submareales (discordancia Huantráiquica) y retransporte de materiales de la subyacente Formación Anacleto. De estos niveles provendrían los restos de Antarctosaurus wichmanianus Huene además de corbículas, tortugas y peces dipnoos. Los niveles psamíticos contienen icnitas de aves y saurópodos, como también corbículas. Se interpreta este ambiente como una llanura subaérea dentro de la plataforma deltaica. Estas facies son correlacionables estratigráficamente con los perfiles de Cinco Saltos donde se hallaron restos de hojas. En Gómez, los miembros medio y superior de la Formación Allen comprenden niveles bentónicos y psamíticos portadores de plesiosaurios, peces, placas de tortugas y pelecípodos salobres, los cuales se atribuyen a un estuario. Para este sector de la cuenca, se observaron dos pulsos transgresivos por el ascenso eustático del mar epicontinental proveniente del Atlántico. Se interpreta la evolución de una plataforma deltaica con el pasaje de una plataforma subácuea en el primer pulso a una llanura subaérea, lo que se representa en el miembro inferior. Un segundo pulso, representado en el miembro medio, conforma este complejo deltaico en un estuario con intervalos de mareas propiciando la colonización de estromatolitos hasta los niveles cuspidales. 


\title{
HUELLAS DE TERÓPODOS AVIANOS DE LA FORMACIÓN ALLEN (CAMPANIANO- MAASTRICHTIANO), PASO CÓRDOBA, PROVINCIA DE RÍO NEGRO, ARGENTINA: IMPLICACIONES ICNOTAXONÓMICAS Y PALEOECOLÓGICAS
}

\author{
R. A. ORTIZ Y Y R. D. JUÁreZ VALIERI 2,3
}

1Rhode 5220, 8332 General Roca, Río Negro, Argentina.paleoroca@yahoo.com.ar

${ }^{2}$ Secretaría de Cultura de la Provincia de Río Negro, Museo Provincial Carlos Ameghino. Belgrano 2150, 8324 Cipolletti, Río Negro, Argentina. rubendjuarez@gmail.com

${ }^{3}$ Museo de Ciencias de Añelo. Calle 6 S/N, 8305 Añelo, Neuquén, Argentina.

El registro icnológico de tetrápodos en el Grupo Malargüe (Cuenca Neuquina) para el intervalo Campaniano superiorMaastrichtiano inferior corresponde a las formaciones equivalentes Loncoche y Allen, que afloran en el sur de la provincia de Mendoza y en Paso Córdoba, provincia de Río Negro, respectivamente. Para esta última localidad de la Formación Allen ya han sido reportados, de modo preliminar, distintos niveles con huellas atribuibles a dinosaurios saurópodos, hadrosaurios y a aves. En el presente estudio se analizan en detalle dichas huellas avianas y ciertas estructuras asociadas. El material de estudio consiste en tres lajas depositadas en el Museo Patagónico de Ciencias Naturales de General Roca (MPCN-RC$N^{\circ} 002$ ). En las mismas se han identificado nueve huellas tridáctilas aisladas y de similar morfología y tamaño (x cm de largo), preservadas en una arenisca de grano fino. Las impresiones de los dígitos son relativamente delgadas y presentan ángulos de apertura de los elementos II-III de $47^{\circ}-65^{\circ}$ y de los elementos III-IV de $35^{\circ}-80^{\circ}$. Estas huellas no muestran evidencias de hallux lo que resulta ser una diferencia con el resto de las icnoespecies cretácicas argentinas, con la excepción de una huella aislada de la Formación Ciénaga del Río Huaco (Maastrichtiano), provincia de San Juan. Por otro lado, es notable la presencia de pequeñas oquedades alrededor de las huellas que son interpretadas como marcas de pico derivadas del hábito alimenticio de estas aves. De esta manera, las huellas estudiadas corresponden a un morfotipo novedoso para el Cretácico Superior de la Cuenca Neuquina.

\section{NUEVAS ASOCIACIONES DE HUELLAS DE DINOSAURIOS EN FACIES CONTINENTALES DE LA FORMACIÓN MULICHINCO (VALANGINIANO TEMPRANO), CUENCA NEUQUINA, ARGENTINA}

\author{
D. Pino ${ }^{1,2}$, R. Gómez ${ }^{1}$, M. Zalazar ${ }^{1}$, I. Díaz Martínez ${ }^{1}$, M. Tunik ${ }^{1}$ y R. A. Coria ${ }^{2}$ \\ IInstituto de investigación en Paleobiología y Geología, UNRN-CONICET. General Roca, Río Negro. Argentina. dapino@unrn.edu.ar \\ ${ }^{2}$ CONICET, Museo Municipal Carmen Funes. Plaza Huincul, Neuquén, Argentina.
}

Se comunica el hallazgo de más de 16 niveles con huellas de dinosaurios a lo largo del perfil Arroyo Salado, provincia de Neuquén, Argentina. Las huellas se hallan en cuerpos tabulares arenosos masivos o con estratificación entrecruzada difusa. El análisis de facies permitió reconocer un paleoambiente fluvial con planicie de inundación bien desarrollada. La presencia de ondulas simétricas de baja amplitud y grietas de desecación, reflejan la generación de cuerpos de agua con fluctuaciones en su columna de agua. La presencia de briznas vegetales y restos de troncos fósiles en heterolitas fangosas indicarían interacción entre estos cuerpos de agua y la desembocadura de ríos. La escasa bioturbación de invertebrados, y la presencia de grietas de sinéresis en algunos niveles, indican estrés y cambios de salinidad en el medio. Las huellas documentadas muestran distintos tipos preservacionales. Algunas huellas circulares a subcirculares están preservadas como moldes naturales, otras huellas tridáctilas lo están como huellas verdaderas con relleno diferencial, y finalmente rastrilladas de huellas tridáctilas y otras subcirculares se encuentran preservadas como subhuellas someras. Teniendo en cuenta, la morfología de las huellas y el patrón de las rastrilladas, se considera que algunas de las mismas fueron impresas por dinosaurios bípedos y otras por cuadrúpedos. Las huellas son más abundantes y diversas en el subambiente de planicie de inundación, constituyendo un lugar propicio para la preservación de las huellas generadas durante la visita o el paso de varios tipos de dinosaurios. 


\title{
IMPLEMENTACIÓN DE PROYECTO ASETUR PARA LA PUESTA EN VALOR DEL PATRIMONIO PALEONTOLÓGICO DE CAMPUS DE LA UNIVERSIDAD NACIONAL DEL COMAHUE, NEUQUÉN CAPITAL
}

\author{
J. D. PORFIRI ${ }^{1,2}$ y D. Dos SANTOS ${ }^{1,2 *}$ \\ ${ }^{1}$ Museo de Ciencias Naturales, Universidad Nacional del Comahue. Buenos Aires 1400, 8300 Neuquén, Neuquén, Argentina. \\ juan.porfiri@central.uncoma.edu.ar; domenica.santos@central.uncoma.edu.ar \\ 2Cátedra de Reptiles Mesozoicos, Facultad de Ingeniería, Universidad Nacional del Comahue. Buenos Aires 1400, 8300 Neuquén, Neuquén, \\ Argentina.
}

El campus de la Universidad Nacional del Comahue, ciudad de Neuquén, cuenta con un valioso registro de vertebrados fósiles. Allí se han rescatado restos de cocodrilos (Notosuchus terrestris, Comahuesuchus brachybuccalis y Neuquensuchus universitas), dinosaurios (Alvarezsaurus calvoi y Velocisaurus unicus, saurópodos titanosaurios), aves (Patagopteryx deferrariisi y Neuquenornis volans), serpientes (Dinilysia patagonica) y huevos de aves enantiornites (con embriones en su interior). Esta diversidad, transforma a esta universidad en el sitio paleontológico más importante de la ciudad. Debido a esta enorme riqueza paleontológica, se está llevando adelante un proyecto ASETUR (2016) denominado "Puesta en valor del área natural protegida (parque universitario) y fortalecimiento del Museo de Ciencias Naturales de la Universidad Nacional del Comahue". Dicho proyecto, en fase de desarrollo, propone valorizar el gran potencial paleontológico que posee el campus de la Universidad Nacional del Comahue. Para ello, se están llevando adelante diversas propuestas administrativas (reglamentos, inventarios, protocolos), adquisición de equipamientos para laboratorio, mejoras en las exhibiciones y digitalización de colecciones. También el acondicionamiento del repositorio del museo posibilita mejorar el estado de resguardo de las colecciones y facilitar el acceso a las mismas a los investigadores. El proyecto también tiene como objetivo brindar un espacio a los visitantes para que puedan valorar la riqueza paleontológica que posee la ciudad de Neuquén. La reapertura al público de este museo, luego de estar cerrado por casi 20 años, permitirá a la sociedad, contar nuevamente con una alternativa en la ciudad de Neuquén para visitar muestras paleontológicas.

*Financiamiento: COFECyT, línea ASETUR 2016.

\section{AÑELO, LA IMPORTANCIA DE NUEVO REPOSITORIO PALEONTOLÓGICO PARA EL CENTRO DE LA PROVINCIA DE NEUQUÉN, PATAGONIA, ARGENTINA}

\author{
J. D. PorfirI ${ }^{1,2,3}$, D. Dos Santos ${ }^{1,2,3}$, R. D. JuÁrez Valieri ${ }^{3,4}$ Y M. Acuña ${ }^{3 *}$ \\ ${ }^{1}$ Museo de Ciencias Naturales, Universidad Nacional del Comahue. Buenos Aires 1400, 8300 Neuquén, Neuquén, Argentina. \\ juan.porfiri@central.uncoma.edu.ar; domenica.santos@central.uncoma.edu.ar \\ ²átedra de Reptiles Mesozoicos, Facultad de Ingeniería, Universidad Nacional del Comahue. Buenos Aires 1400, 8300 Neuquén, Neuquén, \\ Argentina. \\ ${ }^{3}$ Museo de Ciencias de Añelo. Calle 1 y 6 s/n., 8305 Añelo, Neuquén, Argentina \\ 4Secretaría de Cultura de la Provincia de Río Negro, Museo Provincial Carlos Ameghino. Belgrano 2150, 8324 Cipolletti, Río Negro, Argentina. \\ rubendjuarez@gmail.com
}

La localidad de Añelo se encuentra emplazada a unos $90 \mathrm{~km}$ al norte de Neuquén capital. La misma está rodeada de numerosos afloramientos de rocas de edad cretácica, predominantemente del Grupo Neuquén. En estos afloramientos, se han realizado numerosos hallazgos de materiales fósiles, incluyendo importantes especímenes vegetales y animales, entre los que se destacan los dinosaurios. Debido a esto, la Municipalidad de Añelo, FUNYDER y el Museo de Ciencias Naturales de la Universidad Nacional del Comahue, firmaron un convenio para la puesta en marcha de un museo municipal que resguarde el material paleontológico que provenga de la zona centro de la provincia. Este museo, adhiere a la Ley Provincial Nro. 2184/96 de protección de patrimonio Histórico, Arqueológico y Paleontológico, y cuenta con las condiciones de conservación y preservación de materiales paleontológicos y arqueológicos adecuadas. Por este motivo, se busca gestionar la habilitación del museo como un repositorio oficial de colecciones ante la autoridad de aplicación, la Dirección Provincial de Patrimonio Cultural. Esto permitirá que los materiales rescatados en su zona de influencia sean depositados en su repositorio, tal como lo establece la ley citada anteriormente. Uno de los aspectos más relevantes de la creación de este museo, radica en la posibilidad de resguardar el material del área, la cual es intervenida constantemente por las acciones de remoción de suelos que realizan las empresas, debido al notable incremento de la actividad de la industria hidrocarburífera, explotando importantes yacimientos como Vaca Muerta.

*Financiamiento: Municipalidad de Añelo, Neuquén. 


\title{
LA IMPORTANCIA DE LAS ACCIONES EN CONJUNTO PARA POTENCIAR EL DESARROLLO DE LOS MUSEOS: EL “CORREDOR PALEONTOLÓGICO NORTE”
}

\author{
J. D. PorfiRI 1,2,3, D. Dos Santos $1,2,3$, R. D. JuÁrez ValierI ${ }^{3,4}$, C. Banfi ${ }^{5}$ y C. Fuentes ${ }^{6}$
}

${ }^{1}$ Museo de Ciencias Naturales, Universidad Nacional del Comahue. Buenos Aires 1400, 8300 Neuquén, Neuquén, Argentina. juan.porfiri@central.uncoma.edu.ar; domenica.santos@central.uncoma.edu.ar

2Cátedra de Reptiles Mesozoicos, Facultad de Ingeniería, Universidad Nacional del Comahue. Buenos Aires 1400, 8300 Neuquén, Neuquén, Argentina.

${ }^{3}$ Museo de Ciencias de Añelo. Calle 1 y 6 s/n., 8305 Añelo, Neuquén, Argentina.

${ }^{4}$ Secretaría de Cultura de la Provincia de Río Negro, Museo Provincial Carlos Ameghino. Belgrano 2150, 8324 Cipolletti, Río Negro, Argentina. rubendjuarez@gmail.com

${ }_{5}^{5}$ Secretaría de Turismo, Municipalidad de Añelo. Calle 3 Mza 17, lote 3, 8305 Añelo, Neuquén, Argentina. turisanelo@gmail.com

${ }^{6}$ Museo Argentino Urquiza y Parque de Dinosaurios Rinconsaurus. Chos Malal y 30 de Octubre, 8319 Rincón de los Sauces, Neuquén, Argentina. carlosfuentesh@hotmail.com

Dentro de los circuitos turísticos de Neuquén, el recorrido por museos y áreas de interés geopaleontológicos tienen una diversidad y vastedad territorial que su visita requiere varios días. Una forma de gestionar secciones específicas de territorio con sus recursos particulares es generar corredores que inviten a los visitantes realizarlos en tiempos más acotados. A este respecto, la generación de trabajos en conjunto entre tres localidades centrales (Rincón de los Sauces, Añelo y ciudad del Neuquén) proponen considerar la generación del "Corredor Paleontológico Norte" (CPN). Recientemente se ha inaugurado el nuevo Museo Argentino Urquiza y Parque de Dinosaurios Rinconsaurus de Rincón de los Sauces, que permite apreciar numerosas piezas paleontológicas recuperadas en el norte de la meseta patagónica. Por su parte, el Municipio de Añelo en conjunto con la Universidad Nacional del Comahue (UNCO) llevan a cabo la apertura de un nuevo museo centrado en la paleontología propia del centro de la estepa, incluyendo el corazón de Vaca Muerta. Finalmente, el Museo de Ciencias Naturales de la UNCO se encuentra ejecutando una serie de proyectos ASETUR, tendientes a poner en valor de la institución y sus exposiciones paleontológicas. El objetivo actual de estas instituciones es fortalecer vínculos y generar acciones conjuntas para que el CPN pueda centrarse en sus museos y valerse de los servicios de sus localidades, como así también sumar al recorrido los valiosos atractivos existentes en el área, como el Bajo de Añelo, el área natural protegida Auca Mahuida, Tratayén, las bodegas de San Patricio del Chañar, etc.

\section{NEW TITANOSAUR RECORD FROM THE UNIVERSIDAD NACIONAL DEL COMAHUE CAMPUS OF NEUQUÉN CITY (PATAGONIA, ARGENTINA)}

\author{
J. D. Porfiri 1,2,3, R. D. Juárez Valieri ${ }^{3,4}$, A. C. Garrido ${ }^{5,6}$, F. Bellardini 7,8 , and D. Dos Santos $1,2,3$
}

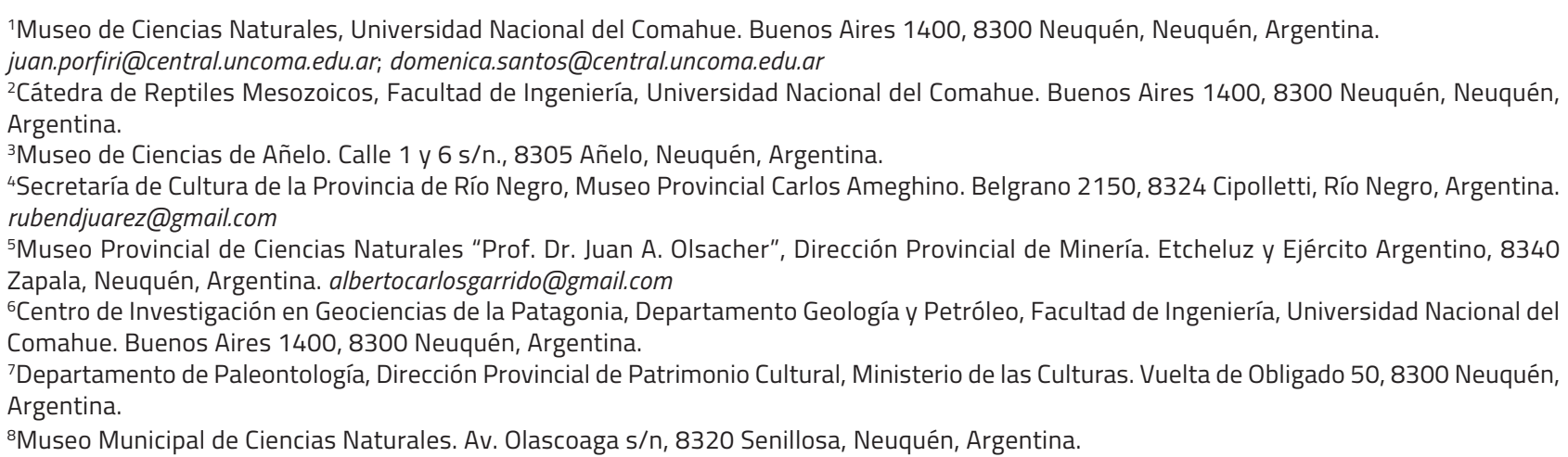

The campus of the Universidad Nacional del Comahue is known for the diverse fauna of small-sized tetrapods that come from the top of the Bajo de la Carpa Formation, however the record of larger dinosaurs is scarce. Here we report a right ulna referred to middle-sized sauropod (MUCPV-1558) that was recently founded in the base of the Anacleto Formation (Neuquén Group: Late Cretaceous). The element is almost complete, only lacking the proximal anterolateral process. The length of the bone is $585 \mathrm{~mm}$, with a robust index barely exceeding 0.4 . In antero-posterior view, the olecranon process is very low, opposite to the condition of most titanosaurs, and the anteromedial process bears a flat articular surface. The distal end is poorly expanded, while the articular surface is subtriangular in-shape in distal view. Several of these characters are shared with different titanosaur taxa, but the combination of characters seen in MUCPv-1558 is shared with Bonitasaura 
salgadoi Apesteguía, which comes from equivalent levels of La Bonita locality, Río Negro Province. Additionally, MUCPv1558 shares with B. salgadoi ulna the distal end morphology, with a concave anteromedial face and a convex posterior face, as well as the development of muscle scar on the anterior face. Although the ulnae are preserved in several titanosaur taxa, this appendicular element is poorly considered in the phylogenetic analysis for bears scarce diagnostic features. Here we refer preliminarly MUCPv-1558 to cf. Bonitasaura, which allows to increase the sauropod fossil record in the southeastern Neuquén basin.

\title{
ANÁLISIS MICROESTRUCTURAL ÓSEO DE FALANGES DE ICTIOSAURIOS JURÁSICOS Y CRETÁCICOS DE CUENCA NEUQUINA
}

\author{
M. TALEVI ${ }^{1,2}$, M. FeRnández ${ }^{1,3}$ Y L. CAMPOS ${ }^{1,3 *}$
}

\begin{abstract}
${ }^{1}$ Consejo Nacional de Investigaciones Científicas y Técnicas (CONICET), Argentina.
${ }^{2}$ Instituto de Investigación en Paleobiología y Geología, Universidad Nacional de Río Negro. Av. Roca 1242, 8332 General Roca, Río Negro, Argentina.mtalevi@unrn.edu.ar

${ }^{3}$ División Paleontología Vertebrados, Unidades de Investigación Anexo Museo, Facultad de Ciencias Naturales y Museo, UNLP. Av. 60 y 122 , 1900 La Plata, Argentina.martafer@fcnym.unlp.edu.ar; Icampos@fcnym.unlp.edu.ar
\end{abstract}

Los ictiosaurios fueron un grupo de reptiles particularmente exitosos en su adaptación secundaria a la vida marina pelágica. Junto con los cetáceos, representan los únicos ejemplos de tetrápodos con patrón corporal semejante al de un pez. Las transformaciones implicaron profundas modificaciones, entre otras, de sus miembros los cuales adquirieron forma de aletas. En el caso particular de los ictiosaurios, la trasformación de los miembros en aletas estuvo acompañada por hiperfalangia e hiperdactilia y, en las formas avanzadas, por el aplanamiento y acortamiento de los huesos zeugopodiales y las falanges, que en muchos casos llegan a ser más anchos que largos. Las falanges, en términos generales, pueden caracterizarse como poligonales, con contornos predominantes rectos y fuertemente empaquetadas entre ellas; o bien predominantemente redondeadas, más espaciadas entre sí. Se ha sugerido que las falanges podrían cambiar de una a otra forma durante la ontogenia. Con el objetivo de explorar si la forma de las falanges está relacionada con distintos tipos de microestructura ósea, se realizaron cortes delgados de falanges de 5 ejemplares de diferentes taxones, MOZ 5803, MOZ 6145, MLP 92-III-21-1 (adultos), MLP 83-XI-15-1, MOZ-PV 6997 (juveniles). La estructura interna los elementos no mostró diferencias histológicas significativas entre las muestras analizadas. En todos los casos, independientemente de la forma o del estadio ontogenético del ejemplar, se observó una región interna de tejido trabecular rodeada por una capa externa de cartílago calcificado. Esto sugiere que la forma poligonal o redondeada de las falanges no estaría relacionada con una variación de la microestructura ósea.

\section{CARACTERIZACIÓN PETROGRÁFICA DEL SEDIMENTO QUE CONTIENE RESTOS DE UN SAURÓPODO PROVENIENTE DEL CAMPUS DE LA UNIVERSIDAD NACIONAL DEL COMAHUE, NEUQUÉN}

\author{
M. A. Tunik ${ }^{1}$, J. PorfirI ${ }^{2,3}$, D. Dos Santos 2,3 , J. I. Ison ${ }^{1}$ y A. C. Garrido 4,5 \\ ${ }^{1}$ Instituto de Investigación en Paleobiología y Geología, Sede Alto Valle, Universidad Nacional de Río Negro. Av. Roca 1242, 8323 Río Negro, \\ Argentina.mtunik@unrn.edu.ar \\ ${ }^{2}$ Museo de Ciencias Naturales, Universidad Nacional del Comahue. Buenos Aires 1400, 8300 Neuquén, Neuquén, Argentina. \\ juan.porfiri@central.uncoma.edu.ar; domenica.santos@central.uncoma.edu.ar \\ ${ }^{3}$ Cátedra de Reptiles Mesozoicos, Facultad de Ingeniería, Universidad Nacional del Comahue. Buenos Aires 1400, 8300 Neuquén, Neuquén, \\ Argentina. \\ "Museo Provincial de Ciencias Naturales "Prof. Dr. Juan A. Olsacher", Dirección Provincial de Minería. Etcheluz y Ejército Argentino, 8340 \\ Zapala, Neuquén, Argentina. albertocarlosgarrido@gmail.com \\ ${ }^{5}$ Centro de Investigación en Geociencias de la Patagonia, Departamento Geología y Petróleo, Facultad de Ingeniería, Universidad Nacional del \\ Comahue. Buenos Aires 1400, 8300 Neuquén, Argentina.
}

Recientemente en el campus de la Universidad Nacional del Comahue, Neuquén, se ha realizado el hallazgo de un nuevo dinosaurio titanosaurio. Se tomaron muestras de la roca que soportaba dichos restos fósiles con el objetivo de presentar la caracterización petrográfica del sedimento. El titanosaurio, fue encontrado en rocas asignadas a la base de la Formación 
Anacleto, a pocos decímetros del contacto con la infrayacente Formación Bajo de la Carpa. La muestra analizada, corresponde a una arenisca gruesa a media, clasificada como litoarenita feldespática (Q74F13L13). Dentro del cuarzo, predomina el tipo monocristalino de extinción recta, siendo el de extinción ondulosa y el policristalino, subordinados. En los feldespatos, predominan las plagioclasas sobre el feldespato alcalino y dentro de los líticos, si bien son porcentajes subordinados, los líticos con texturas granulares, es decir, proveniente de rocas ácidas superan en un 100\% los de origen básico. Tanto los líticos de origen sedimentario, como los metamórficos y los líticos alterados están en porcentajes muy bajos, 2, 0,25 y 1,25\% respectivamente. La muestra presenta un cemento silíceo con dos estadios de crecimiento, pero a la vez, dado la poca consolidación de la muestra, se infiere que la cementación se produjo durante un evento de diagénesis muy temprana. El nivel analizado, ha sido interpretado como canales efímeros. Dichos análisis petrográficos, aportan información sobre la base de la Formación Anacleto en el sector del campus de la Universidad Nacional del Comahue, donde pueden ser frecuentes los hallazgos de vertebrados fósiles.

\title{
ON DICRAEOSAURID (SAUROPODA) VERTEBRA FROM THE LA AMARGA FORMATION (BARREMIAN-APTIAN, LOWER CRETACEOUS), NEUQUÉN BASIN, PATAGONIA, ARGENTINA
}

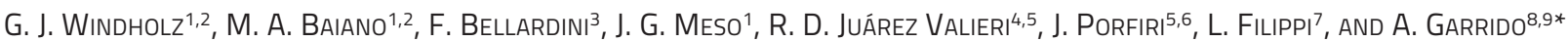 \\ 1CONICET-IIPG, Universidad Nacional de Río Negro. Roca 1242, 8332 Gral. Roca, Río Negro, Argentina. gwindholz@unrn.edu.ar \\ ${ }^{2}$ Museo Carmen Funes. Av. Córdoba 55, 8318 Plaza Huincul, Neuquén, Argentina. \\ ${ }^{3}$ Dpto. de Paleontología, Dirección Provincial de Patrimonio Cultural, Ministerio de las Culturas. Vuelta de Obligado 50, 8300 Neuquén, Provincia \\ del Neuquén, Argentina. \\ ${ }^{4}$ Secretaría de Cultura de la Provincia de Río Negro, Museo Provincial Carlos Ameghino. Belgrano 2150, 8324 Cipolletti, Río Negro, Argentina. \\ ${ }^{5}$ Museo de Ciencias de Añelo. Calle 1 y 6 s/n., 8305 Añelo, Neuquén, Argentina. \\ ${ }^{6}$ Museo de Ciencias Naturales, Universidad Nacional del Comahue. Buenos Aires 1400, 8300 Neuquén, Neuquén, Argentina. \\ ${ }^{7}$ Museo Municipal Argentino Urquiza. Chos Malal s/n., 8319 Rincón de los Sauces, Neuquén, Argentina. \\ ${ }^{8}$ Museo Provincial de Ciencias Naturales "Prof. Dr. Juan A. Olsacher", Dirección Provincial de Minería. Etcheluz y Ejército Argentino, 8340 \\ Zapala, Neuquén, Argentina. albertocarlosgarrido@gmail.com \\ ${ }^{9}$ Centro de Investigación en Geociencias de la Patagonia, Departamento Geología y Petróleo, Facultad de Ingeniería, Universidad Nacional del \\ Comahue. Buenos Aires 1400, 8300 Neuquén, Argentina.
}

Field works carried out by Dr. Bonaparte and collaborators in the La Amarga Formation (Barremian-Aptian) in 2000, allowed the collecting of a vertebral element not previously communicated. This material has not been properly protected, so it is broken and kept in paleontological collection of Museo Provincial "Olsacher" of Zapala city. The descriptions were based solely on a photograph. The parapophyses are located on the dorsal part of the centrum, like in the anterior dorsal vertebrae of Dicraeosaurus hansemanni Janensch and Amargasaurus cazaui Salgado and Bonaparte. The posterior articular surface of the centrum is strongly concave with an elliptical outline. The neural arch bears two deep and wide centropostzygapophyseal fossae with a triangular outline. These fossae are framed laterally by stout centropostzygapophyseal laminae, project dorsoventrally like a massive columnar bone. The transverse processes are inclined dorsally more than $30^{\circ}$ from the horizontal axis, as in the dorsal vertebrae of D. hansemanni, A. cazaui, Brachytrachelopan mesai Rauhut, Remes, Fechner, Cladera and Puerta and Pilmatueia faundezi Coria, Windholz, Ortega and Currie. The element bears an elongate bifid neural spine, character that share with the most of presacral vertebrae of the dicraeosaurids. This structure is straight, dorsoanteriorly oriented and its degree of development reminds to the presacral vertebrae of $A$. cazaui. This element is similar to the anterior dorsal vertebrae of A. cazaui, although it could be comparable with Amargatitanis macni Apesteguía, come from the same locality and lithostratigraphic unit, whose presacral vertebrae are unknown.

*PhD Project registered at Universidad Nacional del Comahue. 
PE-APA 20 (2) - Libro de RESÚMENES

\title{
PRIMER REPORTE DE UN EJEMPLAR JUVENIL DE NEUQUENSAURUS AUSTRALIS (SAUROPODA: TITANOSAURIA)
}

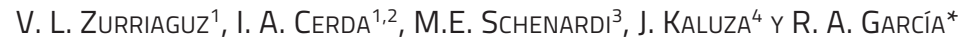 \\ ${ }^{1}$ Instituto de Investigación en Paleobiología y Geología (IIPG) (CONICET-UNRN). Avenida Roca 1242, 3882 General Roca, Río Negro, Argentina. \\ vlzurriaguz@unrn.edu.ar \\ ${ }^{2}$ Museo Carlos Ameghino. Belgrano 1700, Paraje Pichi Ruca (predio Marabunta), 8300 Cipolletti, Río Negro, Argentina. nachocerda6@gmail.com \\ ${ }^{3}$ Universidad Nacional de Río Negro (UNRN). Río Negro, Argentina. \\ "Fundación de Historia Natural "Félix de Azara", Universidad Maimónides. Hidalgo 767 piso 7, 1405 Ciudad Autónoma de Buenos Aires, \\ Argentina.yojonatan@hotmail.com
}

Neuquensaurus australis (Lydekker) es un titanosaurio saltasaurino, proveniente de la Formación Anacleto (Campaniano tardío), cerca de las localidades de Cinco Saltos y Lago Pellegrini, Río Negro. Para esta especie fueron reconocidos dos morfotipos: uno grácil y otro robusto. La forma robusta fue, sin embargo, considerada por algunos autores una especie distinta (Neuquensaurus "robustus"), no existiendo un claro consenso hasta hoy. Aquí presentamos materiales asignables a un ejemplar juvenil de Neuquensaurus australis, integrado por un centro dorsal, un centro caudal anterior, una escápula, un coracoides y una fíbula. Tanto la vértebra caudal como la fíbula poseen los caracteres diagnósticos de la especie (e.g., presencia de fosa ventral no dividida en vértebras caudales y fíbula con una tuberosidad lateral bien desarrollada). La ausencia de neumaticidad en la vértebra, escápula y coracoides indica que dichos caracteres no se expresaban antes de alcanzar un 52\% del tamaño adulto. Por otro lado, a fin de analizar el grado de isometría en el crecimiento, se establecieron proporciones (largo/circunferencia en la porción media) entre la fíbula del ejemplar juvenil y las de dos ejemplares adultos correspondientes tanto al morfotipo grácil como al robusto. El cálculo de proporciones reveló valores (2,91 para el juvenil, 2,99 para el adulto grácil y 2,5 para el robusto), sugiriendo que el ejemplar juvenil se correspondería con este morfotipo. Otra explicación, menos plausible, implica que el juvenil corresponde al morfotipo robusto, el cual experimentaría un drástico cambio en las proporciones óseas durante la segunda mitad del crecimiento.

*Proyecto financiado por PICT 2015-2021.

\section{THE NORTHERNMOST RECORD OF CARCHARODONTOSAURIDAE IN THE NEUQUÉN BASIN}

\author{
J. G. Meso ${ }^{1}$, J. D. Porfiri ${ }^{2,3,4}$, R. D JuÁrez Valieri ${ }^{4,5}$, F. Poblete ${ }^{4}$, And D. Dos Santos $2,3,4$ \\ ${ }^{1}$ CONICET-IIPG, Universidad Nacional de Río Negro. Av. Roca 1242, 8332 Gral. Roca, Río Negro, Argentina. jgmeso@unrn.edu.ar \\ ${ }^{2}$ Museo de Ciencias Naturales, Universidad Nacional del Comahue. Buenos Aires 1400, 8300 Neuquén, Neuquén, Argentina. \\ juan.porfiri@central.uncoma.edu.ar; domenica.santos@central.uncoma.edu.ar \\ ${ }^{3}$ Cátedra de Reptiles Mesozoicos, Facultad de Ingeniería, Universidad Nacional del Comahue. Buenos Aires 1400, 8300 Neuquén, Neuquén, \\ Argentina. \\ 4Museo de Ciencias de Añelo. calle 6 S/N, 8305 Añelo, Neuquén, Argentina. \\ ${ }^{5}$ Secretaria de Cultura de la Provincia de Río Negro, Museo Provincial Carlos Ameghino. Belgrano 2150, 8324 Cipolletti, Río Negro, Argentina. \\ rubendjuarez@gmail.com
}

The fossil record of carcharodontosaurid theropods is frequent in the "middle" Cretaceous of the Neuquén Basin, with three taxa described to date: Giganotosaurus carolinii Coria and Salgado from the Candeleros Formation and Mapusaurus roseae Coria and Currie, and Taurovenator violantei Motta et al. from the overlying Huincul Formation. Additionally, multiple isolated teeth have been reported from these units as also the Portezuelo and Allen formations, expanding the biochron of the clade to the early Maastrichtian. However, several of these assignations has been questioned since the discovery of abelisaurid taxa with superficial similarities in tooth morphology. Here we report an isolated and shed large tooth of theropod (MAÑE 005) from the Aguada Pichana area, located in the center of Neuquén Province. This fossiliferous deposit corresponds to the Candeleros Formation (Neuquén Group). It displays a preserved apicobasal and labiolingual length is 64 and $14 \mathrm{~mm}$, respectively. This element is identified as a lateral tooth based on its general morphology such as strongly compressed laterally, and lenticular to parlinon-shaped cross-section. Through qualitative characteristics, the material has been identified as a dental morphotype corresponding to Carcharodontosauridae. The characteristics that allow this assignation are: arcuate marginal undulations in both surface about distal margin; denticles on the distal carina with an average of 12.5 per mm; braided texture of the enamel; subquadrangular denticles; distal profile of the crown in lateral view weakly concave; mesial carina displaced mesiolabially. Up to now, this discovery constitutes the northermost ocurrence of Carcharodontosauridae in the Neuquén Basin. 